\title{
On the manipulability of approval voting and related scoring rules
}

\author{
Hans Peters · Souvik Roy • Ton Storcken
}

Received: 15 November 2011 / Accepted: 17 November 2011 / Published online: 13 December 2011 (C) The Author(s) 2011. This article is published with open access at Springerlink.com

\begin{abstract}
We characterize all preference profiles at which the approval (voting) rule is manipulable, under three extensions of preferences to sets of candidates: by comparison of worst candidates, best candidates, or by comparison based on stochastic dominance. We perform a similar exercise for $k$-approval rules, where voters approve of a fixed number $k$ of candidates. These results can be used to compare ( $k$-)approval rules with respect to their manipulability. Analytical results are obtained for the case of two voters, specifically, the values of $k$ for which the $k$-approval rule is minimally manipulable - has the smallest number of manipulable preference profiles - under the various preference extensions are determined. For the number of voters going to infinity, an asymptotic result is that the $k$-approval rule with $k$ around half the number of candidates is minimally manipulable among all scoring rules. Further results are obtained by simulation and indicate that $k$-approval rules may improve on the approval rule as far as manipulability is concerned.
\end{abstract}

\section{Introduction}

Approval voting was proposed by Brams and Fishburn (1983) as an alternative to existing election systems, including positional methods, also called scoring rules. So

\footnotetext{
H. Peters $(\varangle) \cdot$ T. Storcken

Department of Quantitative Economics, University of Maastricht, PO Box 616,

6200 MD Maastricht, The Netherlands

e-mail: h.peters@maastrichtuniversity.nl

T. Storcken

e-mail: t.storcken@maastrichtuniversity.nl

S. Roy

University of Caen, Caen, France

e-mail: souvik.roy@unicaen.nl
} 
far, the success of approval voting is at least mixed (cf. Brams and Fishburn 2005). Nevertheless, it receives continued attention and is used, for instance, to select candidates for councils of scientific communities such as the Society for Social Choice and Welfare and the Game Theory Society. The interest in approval voting has recently been confirmed by the publication of the Handbook of Approval Voting (Laslier and Sanver 2010).

In approval voting each voter can approve of as many candidates as he wants. It is well known (Brams and Fishburn 1983, and the references therein) that this procedure is strategy-proof (non-manipulable) if preferences are dichotomous, that is, each voter distinguishes only between a set of good and a set of bad candidates. With more refined preferences, however, strategy-proofness no longer holds. More precisely, a voter will still vote sincerely, i.e., if he approves of a candidate $x$ then he can never do better by not also approving of all candidates preferred to $x$. Such a voter, however, may strategically approve of candidates he would not approve of absent strategic considerations; or not approve of candidates he would approve of absent such considerations. Such voting behavior goes against one of the acclaimed advantages of approval voting, namely that voters should "[...] indicate where they would draw the line between those [candidates] who are acceptable and those who are not" (Brams 2010).

In this paper we study the manipulability of the approval (voting) rule and of a related procedure called $k$-approval (voting) rule. In a $k$-approval rule each voter approves of exactly $k$ candidates. This procedure is less flexible than the approval rule_-voters can provide less information about their preferences-but tends to be also less manipulable, as we will argue. We explicitly use the prudent expression 'tends to' for several reasons. Although the paper presents complete characterizations of the classes of manipulable preference profiles, due to combinatorial complexity comparisons between different voting rules are still largely based on simulations, in particular if the number of voters is larger than two. Also, our results are based on counting (non-)manipulable preference profiles under the 'impartial culture' assumption, and so one has to be careful in applying them to a specific real-life voting situation. Moreover, under $k$-approval voting voters may be forced to vote for unacceptable candidates or not vote for acceptable candidates, a phenomenon which, as we have just discussed, is regarded as strategic behavior under approval voting; on the other hand, with $k$-approval voting the number of approved candidates per voter is under control (namely, fixed), as opposed to approval voting, and moreover $k$ can be chosen optimally in terms of non-manipulability.

An alternative voting rule, related to approval and $k$-approval voting, is to set an upper bound to the number of candidates a voter may approve of. Also this rule may be an interesting modification of approval voting, but in the present paper we choose to compare approval voting to $k$-approval voting as being the positional method (scoring rule in the classical sense as in Young 1975) closest in spirit to the approval rule. A complete comparison in terms of manipulability with other scoring rules (such as Borda count) is outside the scope of this paper. ${ }^{1}$ We do show, however, that

\footnotetext{
$\overline{1}$ Moreover, as Saari (1990) puts it: “[...] it is possible to justify any voting method by choosing an appropriate measure of susceptibility [to manipulation] and imposing the appropriate assumptions on the profiles of voters."
} 
asymptotically (for large electorates) $k$-approval voting with $k$ equal to half of the number of candidates is optimal in terms of non-manipulability.

Preview of the paper In Sect. 2 we introduce the model, necessary notations, and the approval rule and $k$-approval rules. We also define manipulability of these rules. For the approval rule, this implies extending the usual preferences with a cut-off point, indicating which candidates are acceptable and which are not. In Sects. 3 and 4 we characterize the sets of non-manipulable profiles for the approval rule and for $k$-approval rules, respectively. Since these rules are social choice correspondences rather than functions (i.e., can be multi-valued, we need to make assumptions about extending the preferences (weak orderings) of voters over candidates to sets of candidates). We do this in three ways: by comparing the worst candidates of a set, or by comparing the best candidates of a set, or by comparing sets on the basis of stochastic dominance using equal chances. In Sect. 3 we characterize the non-manipulable preference profiles under approval voting for worst, best, and stochastic dominance comparison. The special cases of strict preferences follow as corollaries. Strategy-proofness under dichotomous preferences follows as a special case as well.

In Sect. 4 we characterize the non-manipulable profiles under $k$-approval rules, again for worst, best, and stochastic dominance comparison. We also include a brief consideration of a lexicographic refinement of worst and best comparison. For technical reasons attention in Sect. 4 is restricted to strict preferences.

A main purpose of all these exercises is to compare the approval rule and $k$-approval rules for different values of $k$ with respect to manipulability and under different assumptions about the voters' preferences on sets of candidates. This comparison is based on a simple measure, namely the number of manipulable preference profiles. The implicit assumption is therefore that all profiles are equally likely. This is called 'impartial culture' in the literature. Unfortunately, a complete analytical comparison is out of the question due to the combinatorial complexity of the problem. For this reason, our comparative results are mainly based on simulations and, thus, they are conjectures and suggestions rather than theorems. A selection of the results of these simulations is presented in Sect. 7. They give rise to some prudent conclusions concerning the manipulability of the approval and $k$-approval rules under different assumptions on preference extensions. In particular, they give support to the conjecture that $k$-approval rules for specific values of $k$ may be less susceptible to manipulation than the approval rule.

Nevertheless, we also present some analytical comparison results. In Sect. 5 we consider the two-voter case and compute the optimal $k$ for different preference extensions, that is, the value of $k$ for which the $k$-approval rule is minimally manipulable. For $k=1$, the $k$-approval rule is just plurality voting. In the two-voter case, this is non-manipulable (strategy-proof) under any reasonable preference extension, including those considered in this paper. Plurality voting, however, has a serious drawback. If (the) two voters agree on a good second-ranked candidate but disagree on the first, then under plurality voting this compromise is not chosen; it would be chosen, however, under any other $k$-approval rule. Therefore, for each of the three mentioned preference comparisons and for $k \neq 1$ we have established the overall optimal value of $k$, and the optimal value under the restriction $k \leq m / 2$, where $m$ is the total the number of candidates. The latter restriction is justified by the desirable property of "citizen 
sovereignty': for each candidate there is a preference profile resulting in that candidate as the unique outcome. For $2 \leq k \leq m / 2$ we find $k=2$ as the optimal value in case of best or stochastic dominance comparison, and $k \approx \sqrt{m}$ in case of worst comparison.

On the other extreme, in Sect. 6 we let the number of voters go to infinity and show that even among all scoring rules the $k$-approval rule with $k \in\{(m-1) / 2,(m+1) / 2\}$ if $m$ is odd, and with $k=m / 2$ if $m$ is even, is minimally manipulable. Of course, this result should be interpreted with care, since the probability of manipulability by a single voter is very small anyway if the number of voters is large. The basic intuition for this result is that the (statistical) variance in scores is maximal for the mentioned value(s) of $k$, so that any single voter's probability of being able to change the outcome is minimal.

The paper is concluded by Sect. 8 , in which we draw the main conclusions that can be distilled from the results in the paper.

Further related literature In most voting situations voters have the possibility to manipulate the outcome of the vote by not voting according to their true preferences. The classical theorem of Gibbard (1973) and Satterthwaite (1975) formalizes this fact for social choice functions, which assign a unique candidate to every preference profile, but it also holds for social choice correspondences under various assumptions on preference extensions to sets (e.g., Barberà et al. 2001). The present paper belongs to the strand of literature, initiated by Kelly $(1988,1989)$, which accepts this phenomenon as a matter of fact and looks for social choice rules which are second best in this respect, i.e., least manipulable. Other references include Fristrup and Keiding (1998) and Aleskerov and Kurbanov (1999). Maus et al. (2007) contains a brief overview of this literature.

Of course, counting the non-manipulable profiles is just one way of measuring the degree of (non-)manipulability of voting rules. Many other approaches are possible (e.g., Saari 1990, or recently Campbell and Kelly, 2008). Saari (1990) finds that for the case of three candidates the Borda count is least 'susceptible' to manipulation among all scoring rules. A direct comparison with our results is difficult since we use a different measure of non-manipulability. In fact, for more than three alternatives Saari's results point in the direction of $m / 2$-approval as the best rule, which seems in line with our findings in spite of the differences in model specification.

In this paper we are exclusively concerned with individual manipulation. For coalitional manipulation see, among others, Lepelley and Mbih (1994) and, asymptotically, Pritchard and Wilson (2009). Results of the latter paper are used in Sect. 6. This is not surprising: the $m / 2$-approval rule turns out to be the 'best' rule, at least asymptotically, in many different frameworks, such as indeed coalitional manipulability, but also Condorcet efficiency, i.e, maximizing the probability of choosing a Condorcet winner, or minimizing the probability of choosing a Condorcet loser (Gehrlein and Lepelley 1998; Diss et al. 2010).

\section{Definitions}

We start with some general definitions and notations, and then introduce approval and $k$-approval voting. 


\subsection{General definitions}

The set of voters is $N=\{1, \ldots, n\}$ with $n \geq 2$ and the (finite) set of candidates is $A$ with $|A|=m \geq 3 .^{2}$ A preference is a weak ordering on $A$, i.e., a complete, reflexive, and transitive binary relation on $A$. By $W$ we denote the set of all preferences. A preference is strict (or a linear ordering) if it is antisymmetric, i.e., it cannot contain both $(x, y)$ and $(y, x)$ if $x \neq y$, for all $x, y \in A$. Let $P$ denote the set of all strict preferences on $A$.

A preference profile $w$ is a function from $N$ to $W$, i.e., an element of $W^{N}$. Similarly, a strict preference profile is an element of $P^{N}$. For a preference profile $w, w(i)$ is the preference of voter $i \in N$. For a non-empty subset $B$ of $A, w(i)_{\mid B}$ denotes the restriction of $w(i)$ to the set $B$, i.e., $w(i)_{\mid B}=\{(x, y) \in B \times B \mid(x, y) \in w(i)\}$. Obviously, $w(i)_{\mid A}=w(i)$.

Let $w$ be a preference profile and $i \in N$. Let $1 \leq \ell \leq m$ and suppose there exists a set of candidates $B$ with $|B|=\ell,(x, y) \in w(i)$ and $(y, x) \notin w(i)$ for all $x \in B$ and $y \in A \backslash B$. Then we denote this set by $\beta_{\ell}(w(i))$. Observe that $\beta_{\ell}(w(i))$ exists if and only if there are $\ell$ candidates strictly preferred to the remaining $m-\ell$ candidates according to $w(i)$; that is, $\beta_{\ell}(w(i))$ contains only complete indifference classes of $w(i)$.

Also, for a subset $B$ of $A$, by $\beta\left(w(i)_{\mid B}\right)$ we denote the set of best elements of $B$ according to $w(i)$, that is, $\beta\left(w(i)_{\mid B}\right)=\{x \in B \mid(x, y) \in w(i)$ for all $y \in B\}$. Similarly, $\omega\left(w(i)_{\mid B}\right)$ denotes the set of worst elements of $B$ according to $w(i)$, that is, $\omega\left(w(i)_{\mid B}\right)=\{x \in B \mid(y, x) \in w(i)$ for all $y \in B\}$. The lower contour set of $a \in A$ at $w(i)$ is the set $L(a, w(i))=\{x \in A \mid(a, x) \in w(i)\}$. Observe that $a \in L(a, w(i))$ by reflexivity of $w(i)$.

\subsection{Approval voting}

In approval voting, each voter $i \in N$ approves of $k(i)$ candidates, where $1 \leq k(i) \leq m$ is the choice of the voter. The outcome of the vote is the set of those candidates that receive the largest number of votes. ${ }^{3}$ To formalize this, a report of voter $i$ is a pair $r(i)=(w(i), k(i)) \in W \times\{1, \ldots, m\}$ such that $\beta_{k(i)}(w(i))$ exists. This is then the set of candidates approved of by voter $i$, and it is implied that if a voter approves of a candidate $x$ he also approves of all candidates which are indifferent or strictly preferred to $x$ according to $w(i)$. Note that this is just a convenient way of formalizing approval voting. By $R$ we denote the set of all reports, and by $R^{N}$ the set of all (report) profiles. We denote by

$$
\operatorname{score}(x, r)=\left|\left\{i \in N \mid x \in \beta_{k(i)}(w(i))\right\}\right|
$$

the number of voters who approve of candidate $x \in A$ at profile $r=(w, k)=$ $((w(i), k(i)))_{i \in N} \in R^{N}$. The approval rule $\varphi$, defined by

\footnotetext{
${ }^{2}$ We denote the cardinality of a set $D$ by $|D|$.

${ }^{3}$ Observe that excluding $k(i)=0$ is without loss of generality since the option $k(i)=m$ is available: for the final ranking it will not matter if a voter approves of all or of none of the candidates.
} 


$$
\varphi(r)=\{x \in A \mid \operatorname{score}(x, r) \geq \operatorname{score}(y, r) \text { for all } y \in A\}, \quad r \in R^{N},
$$

assigns to each profile $r$ the subset of candidates with maximal score.

We need a few more notations. For $r=(w, k) \in R^{N}$ and $i \in N, \varphi\left(r_{-i}\right)$ denotes the set of candidates assigned by the approval rule to the restricted profile $r_{-i}=$ $\left(r_{1}, \ldots, r_{i-1}, r_{i+1}, \ldots, r_{n}\right) \in R^{N \backslash\{i\}}$, that is,

$$
\varphi\left(r_{-i}\right)=\left\{x \in A \mid \operatorname{score}\left(x, r_{-i}\right) \geq \operatorname{score}\left(y, r_{-i}\right) \text { for all } y \in A\right\},
$$

where score $\left(x, r_{-i}\right)=\left|\left\{j \in N \backslash\{i\} \mid x \in \beta_{k(j)}(w(j))\right\}\right|$. Finally, for (any) $a \in \varphi\left(r_{-i}\right)$,

$$
\varphi^{-}\left(r_{-i}\right)=\left\{x \in A \mid \operatorname{score}\left(x, r_{-i}\right)=\operatorname{score}\left(a, r_{-i}\right)-1\right\}
$$

is the (possibly empty) set of candidates that have score one less than those of $\varphi\left(r_{-i}\right)$. We call the candidates in $\varphi\left(r_{-i}\right)$ front runners and those in $\varphi^{-}\left(r_{-i}\right)$ serious candidates. ${ }^{4}$ These concepts are convenient in view of the following straightforward observation, which will be used throughout the next section:

$$
\varphi(r)= \begin{cases}\varphi\left(r_{-i}\right) \cap \beta_{k(i)}(w(i)) & \text { if } \varphi\left(r_{-i}\right) \cap \beta_{k(i)}(w(i)) \neq \emptyset \\ \varphi\left(r_{-i}\right) \cup\left[\varphi^{-}\left(r_{-i}\right) \cap \beta_{k(i)}(w(i))\right] & \text { if } \varphi\left(r_{-i}\right) \cap \beta_{k(i)}(w(i))=\emptyset\end{cases}
$$

In order to define (non-)manipulability of the approval rule at particular profiles we need to be able to extend individual preferences to preferences over non-empty subsets of candidates. For a voter $i$ in $N$ and a preference $w(i) \in W$, we say that a binary relation $\succeq_{w(i)}$ on $2^{A} \backslash\{\emptyset\}$ extends $w(i)$ if $\{x\} \succeq_{w(i)}\{y\} \Leftrightarrow(x, y) \in w(i)$ holds for all $x, y \in A$. We write $B \succeq_{w(i)} C$ instead of $(B, C) \in \succeq_{w(i)}$. Also, $\succ_{w(i)}$ and $\sim_{w(i)}$ denote the asymmetric and symmetric parts of $\succeq_{w(i)}$, respectively. ${ }^{5}$

Suppose that $\succeq_{w(i)}$ extends $w(i)$ for all $i \in N$. For $i \in N$ and $r, s \in R^{N}$, we say that $r$ and $s$ are $i$-deviations if $r_{-i}=s_{-i}$. In that case, clearly, $\varphi\left(r_{-i}\right)=\varphi\left(s_{-i}\right)$ and $\varphi^{-}\left(r_{-i}\right)=\varphi^{-}\left(s_{-i}\right)$. The approval rule $\varphi$ is manipulable by voter $i$ at $r=(w, k)$ towards (under preference extension $\succeq_{w(i)}$ ) if $r$ and $s$ are $i$-deviations and $\varphi(s) \succ_{w(i)}$ $\varphi(r)$. The approval rule $\varphi$ is not manipulable at $r$ if for all voters $i$ there is no $i$-deviation $s$ such that $\varphi$ is manipulable by $i$ at $r$ toward $s$.

A few remarks are in order here. First, if $\varphi$ is not manipulable at profile $r=(w, k)$ then implicitly $r$ is assumed to be the true profile. Thus, we assume that each voter $i$ not only has a true preference $w(i)$ but also a true set $\beta_{k(i)}(w(i))$ of candidates which he would approve of absent any strategic considerations. This is similar to the approach in Sanver (2010).

Second, and related, if a voter $i$ manipulates approval voting, under any preference extension considered in this paper he can always do so while still voting sincerely: that is, if $i$ approves of a candidate $x$, he may just as well approve of all candidates which are at least as good as $x$ under the true preference $w(i)$. Hence, manipulation

\footnotetext{
4 Assuming that there is no confusion about the identity of the voter whose vote is left out.

5 In this paper we will consider (three or even more) different ways to extend $w(i)$ over candidates to a binary relation over non-empty sets of candidates.
} 
can take place by only changing the true 'cut off' point $k(i)$ : there is no need to vote insincerely. ${ }^{6}$ Hence, strategic behavior under approval voting does not lead to insincere voting (cf. Brams and Fishburn 1983), but it can lead to approval of 'bad' candidates or disapproval of 'good' candidates, where the cut-off between good and bad is determined by the true number $k(i)$.

We conclude this section with a few examples of manipulation of the approval voting rule. These examples illustrate the different preference extensions to be considered later on in the paper.

Example 2.1 There are six voters $(1, \ldots, 6)$ and four candidates $a, b, c, d$. We consider manipulation by voter 1 and under the approval voting rule it is sufficient to know the total votes cast by the other voters.

- Assume that the votes from 2, . , 6 add up to 4, 4, 3, 2 for $a, b, c, d$, respectively. If voter 1 has preference $c a b \mid d$ (meaning that he (strictly) prefers $c$ over $a$ over $b$ over $d$ and finds the first three acceptable-so $k(1)=3$ in the notation introduced above), then truthful voting results in the winning set $\{a, b\}$. If 1 votes only for $a$ and $c$ then the winning set is $\{a\}$, which is better than $\{a, b\}$ both by comparing the worst elements, and by stochastic comparison: the latter means that if $\{a\}$ is identified with the lottery assigning probability 1 to $a$, and $\{a, b\}$ with lottery assigning equal chances to $a$ and $b$, then the first lottery stochastically dominates the second. If 1 votes only for $c$ then the winning set is $\{a, b, c\}$, which is better by best comparison, i.e., has the best top element.

- Now the votes cast by 2, ., 6 add up to 2, 4, 2, 4 for $a, b, c, d$, respectively, and voter 1 has preference $c a \mid b d$. Truthful voting results in $\{b, d\}$. Voting for $b, a$ and $c$ results in $\{b\}$, which is better by worst and stochastic comparison.

- Finally, the votes cast by 2, ., 6 add up to 3, 4, 2, 2 for $a, b, c, d$, respectively, and voter 1 has preference $c \mid a b d$. Truthful voting results in $\{b\}$. Voting for $a$ and $c$ results in $\{a, b\}$, which is better by best comparison.

Observe that in all these examples voter 1 still votes sincerely, even if he manipulates. This means that he still votes for a top ranked set of candidates. Nevertheless, he may sometimes not vote for a candidate even if he finds that candidate acceptable, or vote for a candidate even if he finds that candidate not acceptable.

\section{$2.3 k$-Approval voting}

A variation on approval voting is obtained by fixing the number of candidates that has to be approved by each voter. Specifically, for a profile $p \in P^{N}$ of strict preferences, a candidate $x \in A$, and a number $k \in\{1, \ldots, m-1\}$, we denote by the $k$-score

$$
\operatorname{score}_{k}(x, p)=\left|\left\{i \in N \mid x \in \beta_{k}(p(i))\right\}\right|
$$

the total number of voters for who candidate $x$ is among the $k$ first ranked candidates at a profile $p$. The $k$-approval rule $\varphi_{k}$, defined by

\footnotetext{
6 We leave it to the reader to verify this claim.
} 


$$
\varphi_{k}(p)=\left\{x \in A \mid \operatorname{score}_{k}(x, p) \geq \operatorname{score}_{k}(y, p) \text { for all } y \in A\right\}, \quad p \in P^{N}
$$

assigns to each profile $p$ the subset of candidates with maximal $k$-score. ${ }^{7}$

Observe that it is, indeed, convenient to restrict attention to strict preferences, since otherwise we might have to split up indifference classes due to the fact that the number of candidates to be approved is now fixed.

The sets $\varphi_{k}\left(p_{-i}\right)$ and $\varphi_{k}^{-}\left(p_{-i}\right)$ of front runners and serious candidates are defined analogously as for the approval rule. Also, completely analogous to (1), we have the following useful observation:

$$
\varphi_{k}(p)= \begin{cases}\varphi_{k}\left(p_{-i}\right) \cap \beta_{k}(p(i)) & \text { if } \varphi_{k}\left(p_{-i}\right) \cap \beta_{k}(p(i)) \neq \varnothing, \\ \varphi_{k}\left(p_{-i}\right) \cup\left[\varphi_{k}^{-}\left(p_{-i}\right) \cap \beta_{k}(p(i))\right] & \text { if } \varphi_{k}\left(p_{-i}\right) \cap \beta_{k}(p(i))=\emptyset\end{cases}
$$

for all $p \in P^{N}, i \in N$, and $1 \leq k \leq m-1$.

The definitions of (non)-manipulability of $\varphi_{k}$ at a profile $p$ are completely analogous to those for the approval rule. In contrast to approval voting, $k$-approval voting may lead to insincere voting since the number of candidates that can be approved of is now fixed.

\section{Manipulability of approval voting}

The purpose of this section is to characterize the (report) profiles at which the approval rule is not manipulable, for three different preference extensions. For the purpose of later comparison with $k$-approval voting we also consider (non-)manipulability under strict preferences. Moreover, we conclude with a brief consideration of dichotomous preferences.

\subsection{Worst comparison}

In this section we extend preferences to sets by considering worst candidates of those sets. Let $i \in N$ and $w(i) \in W$, then we define the extension $\succeq_{w(i)}$ by

$$
B \succeq_{w(i)} C \Leftrightarrow(x, y) \in w(i) \text { for every } x \in \omega\left(w(i)_{\mid B}\right) \text { and } y \in \omega\left(w(i)_{\mid C}\right)
$$

for all non-empty sets $B, C \subseteq A$. Thus, $B$ is weakly preferred to $C$ whenever every worst candidate of $B$ is (weakly) preferred, according to $w(i)$, to every worst candidate of $C$.

Theorem 3.1 Let $r=(w, k) \in R^{N}$. The approval rule $\varphi$ is not manipulable at $r$ under worst comparison if and only if for each voter $i$ at least one of the following two statements holds:

\footnotetext{
7 Unlike the approval rule the $k$-approval rule is a scoring rule in the classical meaning of the word, as in Young (1975); see Sect. 6. Note, further, that the case $k=m$ is uninteresting.
} 
(a) $\varphi\left(r_{-i}\right) \cap \beta_{k(i)}(w(i)) \neq \varnothing$ and $\{x\} \sim_{w(i)} \quad\{y\}$ for all $x, y \in \varphi\left(r_{-i}\right) \cap$ $\beta_{k(i)}(w(i))$.

(b) $\{x\} \sim_{w(i)}\{y\}$ for all $x, y \in \varphi\left(r_{-i}\right)$.

In words, condition (a) requires that if among the front runners there are candidates belonging to the $k(i)$ highest ranked candidates of voter $i$, then $i$ is indifferent between those candidates; and (b) requires that voter $i$ is indifferent between all front runners.

Proof of Theorem 3.1 For the if-part, let $s$ be an $i$-deviation of $r$.

In case (a), it follows by (1) that $\varphi(r)=\varphi\left(r_{-i}\right) \cap \beta_{k(i)}(w(i))$. By the assumption in (a), $\varphi(r)=\beta\left(\left.w(i)\right|_{\varphi\left(r_{-i}\right)}\right)$. Again by (1), $\varphi(s) \cap \varphi\left(r_{-i}\right) \neq \emptyset$, so for every $x \in \varphi(r)=\beta\left(\left.w(i)\right|_{\varphi\left(r_{-i}\right)}\right)$ it follows that $\{x\} \succeq_{w(i)} \varphi(s)$. So, $\varphi(r) \succeq_{w(i)} \varphi(s)$.

Now consider case (b) and assume $\varphi\left(r_{-i}\right) \cap \beta_{k(i)}(w(i))=\emptyset$ otherwise we are done by (a). Then $\varphi\left(r_{-i}\right) \subseteq \varphi(r)$, so we have $\omega\left(w(i)_{\mid \varphi(r)}\right)=\varphi\left(r_{-i}\right)$. Since, by (1), $\varphi(s) \cap \varphi\left(r_{-i}\right) \neq \emptyset$, we have again $\varphi(r) \succeq_{w(i)} \varphi(s)$.

For the only if-part, suppose that there is an voter $i$ for whom (a) nor (b) holds. It is sufficient to prove that $\varphi$ is manipulable at profile $r$ by voter $i$. Observe that either there exist $x, y \in \varphi\left(r_{-i}\right) \cap \beta_{k(i)}(w(i))$ such that $\{x\} \succ_{w(i)}\{y\}$, or $\varphi\left(r_{-i}\right) \cap \beta_{k(i)}(w(i))=\varnothing$ and there exist $x, y \in \varphi\left(r_{-i}\right)$ such that $\{x\} \succ_{w(i)}\{y\}$. In both cases, by (1), $x, y \in$ $\varphi(r)$. Now consider the report $s(i)=\left(w^{\prime}(i), 1\right)$ of voter $i$ such that $\beta\left(w^{\prime}(i)\right)=\{x\}$. Then, by (1) again, $\varphi(s)=\{x\} \succ_{w(i)} \varphi(r)$.

We now consider the subclass of strict preferences. This will enable us to compare approval voting to $k$-approval voting, which is studied in the next section.

\subsubsection{Strict preferences}

The following result considers manipulability of the approval rule $\varphi$ when preferences are strict. Let $S^{N}$ denote the set of all reports $(w, k) \in R^{N}$ such that $w \in P^{N}$.

Corollary 3.2 Let $r=(w, k) \in S^{N}$. The approval rule $\varphi$, restricted to $S^{N}$, is not manipulable at $r$ under worst comparison if and only if for each voter $i$ at least one of the following two statements holds:

(a) $\left|\varphi\left(r_{-i}\right) \cap \beta_{k(i)}(w(i))\right|=1$.

(b) $\left|\varphi\left(r_{-i}\right)\right|=1$.

Proof For the only-if direction, note that if voter $i$ can manipulate via a preference in $W$, then $i$ can also manipulate by a strict preference, by strictifying the weak preference in any arbitrary way. Thus, the only-if direction follows from Theorem 3.1. The if-direction is immediate from Theorem 3.1.

\subsection{Best comparison}

In this section we extend preferences to sets by considering best candidates of those sets. Let $i \in N$ and $w(i) \in W$, then we define the extension $\succeq_{w(i)}$ by $^{8}$

\footnotetext{
${ }^{8}$ In order to avoid cumbersome notation we will use the same symbols for different preference extensions in this paper.
} 


$$
B \succeq_{w(i)} \quad C \Leftrightarrow(x, y) \in w(i) \text { for every } x \in \beta\left(w(i)_{\mid B}\right) \text { and } y \in \beta\left(w(i)_{\mid C}\right)
$$

for all non-empty sets $B, C \subseteq A$. Thus, $B$ is weakly preferred to $C$ whenever every best candidate of $B$ is (weakly) preferred, according to $w(i)$, to every best candidate of $C$.

Theorem 3.3 Let $r=(w, k) \in R^{N}$. The approval rule $\varphi$ is not manipulable at $r$ under best comparison if and only if for each voter $i$ at least one of the following two statements holds:

(a) $(x, y) \in w(i)$ for all $x \in \beta\left(w(i)_{\mid \varphi\left(r_{-i}\right)}\right)$ and all $y \in \varphi^{-}\left(r_{-i}\right)$.

(b) $\varphi\left(r_{-i}\right) \cap \beta_{k(i)}(w(i))=\emptyset$ and $\varphi^{-}\left(r_{-i}\right) \cap \beta_{k(i)}(w(i)) \neq \emptyset$.

In words, condition (a) requires that any best candidate among the front runners is preferred by $i$ over all serious candidates; and (b) requires that none of the front runners is among his $k(i)$ highest ranked candidates, but some of the serious candidates are among his $k(i)$ highest ranked candidates.

Proof of Theorem 3.3 For the if-part, let $s$ be an $i$-deviation of $r$.

In case (a), it follows by (1) that there exists $x \in \varphi(r)$ with $x \in \beta\left(w(i)_{\mid \varphi\left(r_{-i}\right)}\right)$. So by (a), $\{x\} \succeq_{w(i)}\{y\}$ for all $y \in \varphi\left(r_{-i}\right) \cup \varphi^{-}\left(r_{-i}\right)$. This implies $\varphi(r) \succeq_{w(i)} \varphi(s)$.

In case (b), by (1), $\varphi(r)=\varphi\left(r_{-i}\right) \cup\left[\varphi^{-}\left(r_{-i}\right) \cap \beta_{k(i)}(w(i))\right]$. So $\varphi(r) \succeq_{w(i)}\{x\}$ for all $x \in \varphi\left(r_{-i}\right) \cup \varphi^{-}\left(r_{-i}\right)$. This implies again $\varphi(r) \succeq_{w(i)} \varphi(s)$.

For the only if-part, suppose that there is an voter $i$ for whom (a) nor (b) holds. It is sufficient to prove that $\varphi$ is manipulable at profile $r$ by voter $i$. Observe that either (i) $\varphi\left(r_{-i}\right) \cap \beta_{k(i)}(w(i)) \neq \emptyset$ and there exists $y \in \varphi^{-}\left(r_{-i}\right)$ such that $\{y\} \succ_{w(i)}$ $\beta\left(w(i)_{\mid \varphi\left(r_{-i}\right)}\right)$; or (ii) $\varphi^{-}\left(r_{-i}\right) \cap \beta_{k(i)}(w(i))=\emptyset$ and there exists $y \in \varphi^{-}\left(r_{-i}\right)$ such that $\{y\} \succ_{w(i)} \beta\left(w(i)_{\mid \varphi\left(r_{-i}\right)}\right)$. Note that, in both cases, $\varphi(r) \subseteq \varphi\left(r_{-i}\right)$. For both cases, consider the report $s(i)=\left(w^{\prime}(i), 1\right)$ of voter $i$ such that $\beta\left(w^{\prime}(i)\right)=\{y\}$. Then by (1), $\varphi(s)=\varphi\left(r_{-i}\right) \cup\{y\}$, which implies $\varphi(s) \succ_{w(i)} \varphi(r)$.

For strict preferences we have the following corollary. The proof is straightforward and therefore omitted.

Corollary 3.4 Let $r=(w, k) \in S^{N}$. The approval rule $\varphi$, restricted to $S^{N}$, is not manipulable at $r$ under best comparison if and only if for each voter $i$ at least one of the following two statements holds:

(a) $(x, y) \in w(i)$ for all $y \in \varphi^{-}\left(r_{-i}\right)$, where $\{x\}=\beta\left(w(i)_{\mid \varphi\left(r_{-i}\right)}\right)$.

(b) $\varphi\left(r_{-i}\right) \cap \beta_{k(i)}(w(i))=\emptyset$ and $\varphi^{-}\left(r_{-i}\right) \cap \beta_{k(i)}(w(i)) \neq \emptyset$.

\subsection{Stochastic dominance comparison}

In this section comparisons of sets of candidates are based on stochastic dominance. To formalize this we need some further notions. Let $u$ be a function from $A$ to $\mathbb{R}$. Then $u$ is said to be a utility function representing preference $w(i)$ of voter $i$, if for all candidates $x$ and $y$ in $A$

$$
(x, y) \in w(i) \text { if and only if } u(x) \geq u(y)
$$


Let $B$ and $C$ be two nonempty subsets of candidates. Voter $i$ is said to prefer $B$ to $C$ according to stochastic dominance at preference $w(i)$, denoted as $B \succeq_{w(i)} C$, if

$$
\sum_{a \in B} \frac{1}{|B|} u(a) \geq \sum_{a \in C} \frac{1}{|C|} u(a) \text { for every utility function } u \text { representing } w(i) \text {. }
$$

This preference extension ${ }^{9}$ is based on the idea that, if we attach equal probabilities to the candidates in each set, then the expected utility of the resulting lottery over $B$ should be at least as high as the expected utility of the resulting lottery over $C$, for each utility function representing $p(i)$. Clearly, and in contrast to worst and best comparison in the preceding sections, this preference extension is not complete: many sets are incomparable. Observe that our notion of manipulability implies that a voter manages to obtain a preferred and thus comparable set.

In the following theorem we characterize the non-manipulable profiles under the stochastic dominance preference extension. To understand the proof, it is sometimes convenient to keep in mind the familiar characterization (or definition) of stochastic dominance involving only probabilities. This characterization says that a lottery $\ell$ is preferred over another lottery $\ell^{\prime}$ if it can be obtained by shifting probability in $\ell^{\prime}$ to preferred candidates.

Theorem 3.5 Let $r=(w, k) \in R^{N}$. The approval rule $\varphi$ is not manipulable at $r$ under stochastic dominance if and only iffor each voter $i$ at least one of the following three statements holds:

(a) $\varphi\left(r_{-i}\right) \subseteq\left[A \backslash \beta_{k(i)}(w(i))\right]$ and $\varphi^{-}\left(r_{-i}\right) \cap \beta_{k(i)}(w(i)) \neq \emptyset$.

(b) $\varphi\left(r_{-i}\right) \cap \beta_{k(i)}(w(i)) \neq \emptyset$ and $\{x\} \sim_{w(i)}\{y\}$ for all $x, y \in \varphi\left(r_{-i}\right) \cap \beta_{k(i)}(w(i))$ and $\left[A \backslash \beta_{k(i)}(w(i))\right] \cap \varphi\left(r_{-i}\right) \neq \emptyset$.

(c) $\{x\} \sim_{w(i)}\{y\}$ for all $x, y \in \varphi\left(r_{-i}\right)$ and $\varphi^{-}\left(r_{-i}\right) \subseteq L(x, w(i))$ for some $x \in \varphi\left(r_{-i}\right)$.

In words, these three cases can be described as follows. In case (a), no front runner but at least one serious candidate belongs to the $k(i)$ highest ranked candidates. In case (b) there are front runners among the $k(i)$ highest ranked candidates and voter $i$ is indifferent between them, but there are also lower ranked front runners. In case (c) voter $i$ is indifferent between the front runners, and all serious candidates are lower ranked than some of the front runners.

For a proof of this theorem see the Appendix.

The following corollary (proof omitted) applies to strict preferences.

Corollary 3.6 Let $r=(w, k) \in S^{N}$. The approval rule $\varphi$, restricted to $S^{N}$, is not manipulable at $r$ under stochastic dominance comparison if and only if for each voter $i$ at least one of the following three statements holds:

(a) $\varphi\left(r_{-i}\right) \subseteq\left[A \backslash \beta_{k(i)}(w(i))\right]$ and $\varphi^{-}\left(r_{-i}\right) \cap \beta_{k(i)}(w(i)) \neq \emptyset$.

(b) $\varphi\left(r_{-i}\right) \cap \beta_{k(i)}(w(i))=\{x\}$ for some $x \in A$ and $\left[A \backslash \beta_{k(i)}(w(i))\right] \cap \varphi\left(r_{-i}\right) \neq \emptyset$.

(c) $\varphi\left(r_{-i}\right)=\{x\}$ for some $x \in A$ and $\varphi^{-}\left(r_{-i}\right) \subseteq L(x, w(i))$.

$\overline{9}$ The stochastic dominance criterion to compare sets has been used before, see e.g. Barberà et al. (2001). 


\subsection{Dichotomous preferences}

A preference $w(i) \in W$ is dichotomous if it has two indifference classes, i.e., there are disjoint subsets $B_{1} \neq \emptyset$ and $B_{2}$ of $A$ such that $A=B_{1} \cup B_{2},(x, y),(y, x) \in w(i)$ for all $x, y \in B_{1}$ and for all $x, y \in B_{2}$, and $(x, y) \in w(i),(y, x) \notin w(i)$ for all $x \in B_{1}$ and $y \in B_{2}$. Let $D \subseteq W$ denote the subset of all dichotomous preferences. A report $r(i)=(w(i), k(i))$ is in $R_{d}$ if $w(i)$ is dichotomous and $k(i)$ is the cardinality of the higher indifference class of $w(i)$, i.e., $k(i)=\left|B_{1}\right|$ in the notation above. ${ }^{10}$ A report $r(i) \in R_{d}$ is called dichotomous as well. In the following corollary we show that the approval rule is strategy-proof when restricted to dichotomous report profiles, under all three preference extensions considered in this paper: this means that $\varphi$ is manipulable at no $r \in R_{d}^{N}$ under any of these preference extensions. This result confirms well known results on approval voting, see Brams and Fishburn (1983) and the references therein. For completeness, a proof based on the earlier results in this section is given in the Appendix.

Corollary 3.7 The approval rule $\varphi$, restricted to $R_{d}^{N}$, is strategy-proof under the worst, best, and stochastic dominance preference extensions.

\section{Manipulability of $k$-approval voting}

In this section we characterize the profiles of preferences at which the $k$-approval rule is not manipulable for different preference extensions, starting with worst, best, and stochastic dominance comparison.

\subsection{Worst comparison}

For the definition of the worst comparison preference extension see Sect. 3.1.

The following theorem characterizes all profiles at which the $k$-approval rule is not manipulable under worst comparison.

Theorem 4.1 Let $p \in P^{N}$. The $k$-approval rule $\varphi_{k}$ is not manipulable at $p$ under worst comparison if and only if for each voter $i$ at least one of the following three statements holds:

(a) $\left|\varphi_{k}\left(p_{-i}\right) \cap \beta_{k}(p(i))\right|=1$.

(b) $\left|\varphi_{k}\left(p_{-i}\right)\right|=1$.

(c) $A \backslash \beta_{k}(p(i)) \subsetneq \varphi_{k}\left(p_{-i}\right)$.

In words, condition (a) requires that exactly one of that voter $i$ 's $k$ highest ranked candidates is a front runner; (b) requires that there is a unique front runner; and (c) requires that the front runners are a strict subset of the $m-k$ lowest ranked candidates.

Proof of Theorem 4.1 For the if-part, let $i \in N$ and let $q$ be an $i$-deviation of $p$. Assume that at least one of the cases (a), (b), and (c) holds. We show that voter $i$ cannot manipulate from $p$ to $q$.

$\overline{10 \text { Observe that, in this case, } k(i)}$ is uniquely determined by $w(i)$. 
In case (a), let $\{x\}=\varphi_{k}\left(p_{-i}\right) \cap \beta_{k}(p(i))$. By (2), $\varphi_{k}(p)=\{x\}$. Again by (2), either $\varphi_{k}(q) \subseteq \varphi_{k}\left(p_{-i}\right)$ or $\varphi_{k}\left(p_{-i}\right) \subseteq \varphi_{k}(q)$. In the first case, if $x \in \varphi_{k}(q)$, then $\varphi_{k}(p)=$ $\{x\} \succeq_{p(i)} \varphi_{k}(q)$; if $x \notin \varphi_{k}(q)$ then $\varphi_{k}(q) \subseteq A \backslash \beta_{k}(p(i))$ so that again $\varphi_{k}(p)=$ $\{x\} \succeq_{p(i)} \varphi_{k}(q)$. In the second case, $\varphi_{k}(p)=\{x\} \subseteq \varphi_{k}(q)$, hence $\varphi_{k}(p) \succeq_{p(i)} \varphi_{k}(q)$.

In case (b), let $\varphi_{k}\left(p_{-i}\right)=\{x\}$ for some candidate $x$. If $x \in \beta_{k}(p(i))$ we are done by case (a). If $x \notin \beta_{k}(p(i))$ then by (2), $\varphi_{k}(p)=\{x\} \cup\left[\varphi_{k}^{-}\left(p_{-i}\right) \cap \beta_{k}(p(i))\right]$ and, thus, $\omega\left(p(i)_{\mid \varphi_{k}(p)}\right)=x$. Further, also by (2), $\varphi_{k}(q)=\{x\}$ or $\varphi_{k}(q)=\{x\} \cup$ $\left[\varphi_{k}^{-}\left(p_{-i}\right) \cap \beta_{k}(q(i))\right]$; in both cases, $\left(x, \omega\left(p(i)_{\mid \varphi_{k}(q)}\right)\right) \in p(i)$ and, thus, $\varphi_{k}(p) \succeq_{p(i)}$ $\varphi_{k}(q)$.

In case (c), by (2) we have $\varphi_{k}(p)=\varphi_{k}\left(p_{-i}\right) \cap \beta_{k}(p(i))$ and $\varphi_{k}(q)=\varphi_{k}\left(q_{-i}\right) \cap$ $\beta_{k}(q(i))=\varphi_{k}\left(p_{-i}\right) \cap \beta_{k}(q(i))$. If $\beta_{k}(q(i))=\beta_{k}(p(i))$ then $\varphi_{k}(p)=\varphi_{k}(q)$. Otherwise, since $A \backslash \beta_{k}(p(i)) \subsetneq \varphi_{k}\left(p_{-i}\right)$, there is a $y \in\left[A \backslash \beta_{k}(p(i))\right] \cap \varphi_{k}(q)$. Hence, $\varphi_{k}(p) \succeq_{p(i)} \varphi_{k}(q)$.

For the only-if part, suppose that there is a voter $i \in N$ such that none of the three cases (a), (b), and (c) holds. It is sufficient to prove that $\varphi_{k}$ is manipulable at profile $p$ by voter $i$. For this, in turn, it is sufficient to prove that $i$ can manipulate at profile $p$ for the following two cases.

Case (i): $\varphi_{k}\left(p_{-i}\right) \cap \beta_{k}(p(i))=\emptyset$ and $\left|\varphi_{k}\left(p_{-i}\right)\right| \geq 2$.

Let $b=\beta\left(p(i)_{\mid \varphi_{k}\left(p_{-i}\right)}\right)$. Take $q(i)$ such that the positions in $p(i)$ of $b$ and one of the candidates in $\beta_{k}(p(i))$ are swapped. Then $\varphi_{k}(q)=\{b\}$ and $\varphi_{k}(q) \succ_{p(i)} \varphi_{k}(p)$, hence voter $i$ can manipulate at profile $p$ toward $q$.

Case (ii): $\left|\varphi_{k}\left(p_{-i}\right) \cap \beta_{k}(p(i))\right| \geq 2$ and $\left[A \backslash \beta_{k}(p(i))\right] \nsubseteq \varphi_{k}\left(p_{-i}\right)$.

Let $w=\omega\left(p(i)_{\mid \varphi_{k}\left(p_{-i}\right) \cap \beta_{k}(p(i))}\right)$ and $y \in A \backslash\left[\beta_{k}(p(i)) \cup \varphi_{k}\left(p_{-i}\right)\right]$. Let $q(i)$ be obtained from $p(i)$ by swapping the positions of the candidates $w$ and $y$. By $(2), \varphi_{k}(p)=\varphi_{k}\left(p_{-i}\right) \cap \beta_{k}(p(i))$ and $\varphi_{k}(q)=$ $\varphi_{k}\left(p_{-i}\right) \cap \beta_{k}(p(i)) \backslash\{w\}$ it follows that $\varphi_{k}(q) \succ_{p(i)} \varphi_{k}(p)$, proving that $\varphi_{k}$ is manipulable by voter $i$ at profile $p$ toward $q$.

\subsection{Best comparison}

For the definition of the best comparison preference extension see Sect. 3.2.

The following theorem characterizes all profiles at which the $k$-approval rule is not manipulable under best comparison.

Theorem 4.2 Let $p \in P^{N}$. The k-approval scoring rule $\varphi_{k}$ is not manipulable at $p$ under best comparison if and only iffor each voter $i$ at least one of the following three statements holds:

(a) $\left(\beta\left(p(i)_{\mid \varphi_{k}\left(p_{-i}\right)}\right), x\right) \in p(i)$ for all $x \in \varphi_{k}^{-}\left(p_{-i}\right)$.

(b) $\varphi_{k}\left(p_{-i}\right) \cap \beta_{k}(p(i))=\emptyset$ and $\varphi_{k}^{-}\left(p_{-i}\right) \cap \beta_{k}(p(i)) \neq \emptyset$.

(c) $\left|\varphi_{k}\left(p_{-i}\right) \cap \beta_{k}(p(i))\right|>\left|A \backslash\left[\beta_{k}(p(i)) \cup \varphi_{k}\left(p_{-i}\right)\right]\right|$.

In words, condition (a) requires that the best candidate among the front runners is preferred over all serious candidates; (b) requires that no front runner is among his $k$ 
first ranked candidates, but some of the serious candidates are; and (c) requires that the number of the voter's $k$ highest ranked candidates among the front runners is larger than the number of candidates that are neither among his $k$ highest ranked nor among the front runners.

Proof of Theorem 4.2 For the if-part, let $q$ be an $i$-deviation of $p$. Note that $\varphi_{k}\left(p_{-i}\right)=\varphi_{k}\left(q_{-i}\right)$ and $\varphi_{k}^{-}\left(p_{-i}\right)=\varphi_{k}^{-}\left(q_{-i}\right)$. Assume that at least one of the cases (a), (b), and (c) holds. We show that voter $i$ cannot manipulate from $p$ to $q$.

In case (a), for both cases occurring in (2), we obtain $\beta\left(p(i)_{\mid \varphi_{k}(p)}\right)=$ $\beta\left(p(i)_{\mid \varphi_{k}\left(p_{-i}\right)}\right)$. Since $\beta\left(p(i)_{\mid \varphi_{k}(q)}\right) \in \varphi_{k}\left(p_{-i}\right) \cup \varphi_{k}^{-}\left(p_{-i}\right)$ and by the assumption for case (a), we conclude that $\varphi_{k}(p) \succeq_{p(i)} \varphi_{k}(q)$.

In case (b), again using (2), we have $\varphi_{k}(p)=\varphi_{k}\left(p_{-i}\right) \cup\left[\varphi_{k}^{-}\left(p_{-i}\right) \cap \beta_{k}(p(i))\right]$, hence $\beta\left(p(i)_{\mid \varphi_{k}(p)}\right)=\beta\left(p(i)_{\mid \varphi_{k}^{-}\left(p_{-i}\right) \cap \beta_{k}(p(i))}\right)$; and $\varphi_{k}(q) \in \varphi_{k}\left(p_{-i}\right) \cup\left[\varphi_{k}^{-}\left(p_{-i}\right) \cap\right.$ $\left.\beta_{k}(q(i))\right]$. By the assumptions for this case, $\varphi_{k}(p) \succeq_{p(i)} \varphi_{k}(q)$.

In case (c), it is easy to see that $\left|A \backslash \varphi_{k}\left(p_{-i}\right)\right|<\left|\beta_{k}(p(i))\right|=k=\left|\beta_{k}(q(i))\right|$, hence $\beta_{k}(q(i)) \cap \varphi_{k}\left(p_{-i}\right) \neq \emptyset$. Therefore, by (2) we have $\varphi_{k}(p)=\varphi_{k}\left(p_{-i}\right) \cap \beta_{k}(p(i))$ and $\varphi_{k}(q)=\varphi_{k}\left(p_{-i}\right) \cap \beta_{k}(q(i)) \subseteq \varphi_{k}\left(p_{-i}\right)$. Thus, also in this case $\varphi_{k}(p) \succeq_{p(i)} \varphi_{k}(q)$.

For the only-if part, suppose that there is a voter $i \in N$ such that none of the three cases (a), (b), and (c) holds. It is sufficient to prove that $\varphi_{k}$ is manipulable at profile $p$ by voter $i$. For this, in turn, it is sufficient to prove that $i$ can manipulate at profile $p$ for the following two cases.

Case (i): There is an $x \in \varphi_{k}^{-}\left(p_{-i}\right)$ such that $(x, b) \in p(i)$, where $b=\beta\left(\varphi_{k}\left(p_{-i}\right)\right.$, $\left.p(i)_{\mid \varphi_{k}\left(p_{-i}\right)}\right) ; \varphi_{k}\left(p_{-i}\right) \cap \beta_{k}(p(i)) \neq \emptyset$; and $\left|\varphi_{k}\left(p_{-i}\right) \cap \beta_{k}(p(i))\right| \leq$ $\left|A \backslash\left[\beta_{k}(p(i)) \cup \varphi_{k}\left(p_{-i}\right)\right]\right|$.

For this case, note that $x \in \beta_{k}(p(i))$. By the assumptions for this case we can take a $q(i) \in P$ with $x \in \beta_{k}(q(i))$ and $\varphi_{k}\left(p_{-i}\right) \cap \beta_{k}(q(i))=\emptyset$. Hence, $x \in \varphi_{k}(q) \backslash \varphi_{k}(p)$ and, thus, $\varphi_{k}(q) \succ_{p(i)} \varphi_{k}(p)$. So $i$ can manipulate at profile $p$ toward $q$.

Case (ii): There is an $x \in \varphi_{k}^{-}\left(p_{-i}\right)$ such that $(x, b) \in p(i)$, where $b=$ $\beta\left(p(i)_{\mid \varphi_{k}\left(p_{-i}\right)}\right)$; and $\varphi_{k}^{-}\left(p_{-i}\right) \cap \beta_{k}(p(i))=\emptyset$.

In this case, $\varphi_{k}(p)=\varphi_{k}\left(p_{-i}\right)$. Note that the sets $\beta_{k}(p(i)), \varphi_{k}\left(p_{-i}\right)$, and $\varphi_{k}^{-}\left(p_{-i}\right)$ are pairwise disjoint. So we can take $q(i) \in P$ such that $x \in \beta_{k}(q(i))$ and $\varphi_{k}\left(p_{-i}\right) \cap \beta_{k}(q(i))=\emptyset$. Then $\varphi_{k}(q) \supseteq \varphi_{k}\left(p_{-i}\right) \cup\{x\}$, so $x \in \varphi_{k}(q) \backslash \varphi_{k}(p)$, thus $\varphi_{k}(q) \succ_{p(i)} \varphi_{k}(p)$ and $i$ can manipulate at profile $p$ toward $q$.

\subsection{Stochastic dominance comparison}

For the definition of the stochastic dominance comparison preference extension see Sect. 3.3.

The following theorem characterizes all profiles at which the $k$-approval rule is not manipulable under stochastic dominance comparison. Its proof is placed in the Appendix. 
Theorem 4.3 Let $p \in P^{N}$. The $k$-approval scoring rule $\varphi_{k}$ is not manipulable at $p$ under stochastic dominance comparison if and only if for all voters $i$ at least one of the following five statements holds:

(a) $A \backslash \beta_{k}(p(i)) \subsetneq \varphi_{k}\left(p_{-i}\right)$.

(b) $\varphi_{k}\left(p_{-i}\right) \subseteq\left[A \backslash \beta_{k}(p(i))\right]$ and $\varphi_{k}^{-}\left(p_{-i}\right) \cap \beta_{k}(p(i)) \neq \emptyset$.

(c) $\varphi_{k}\left(p_{-i}\right) \cap \beta_{k}(p(i))=\{w\}$ for some $w \in A$ and $\left[A \backslash \beta_{k}(p(i))\right] \cap \varphi_{k}\left(p_{-i}\right) \neq \emptyset$.

(d) $\varphi_{k}\left(p_{-i}\right)=\{w\}$ for some $w \in A$ and $\varphi_{k}^{-}\left(p_{-i}\right) \subseteq L(w, p(i))$.

(e) $\varphi_{k}\left(p_{-i}\right)=\{w\}$ for some $w \in A$ and $\left|\varphi_{k}^{-}\left(p_{-i}\right) \cap L(w, p(i))\right|>m-k$.

In words, these five cases can be described as follows. In case (a), at least one of voter $i$ 's $k$ highest ranked candidates and all of his lower ranked candidates are front runners. In case (b), no front runner but at least one serious candidate is among his $k$ highest ranked candidates. In case (c) there is a unique front runner among voter $i$ 's $k$ highest ranked candidates, but there are lower ranked front runners as well. In case (d) there is a unique front runner, which is preferred by $i$ to all serious candidates. In case (e) there is again a unique front runner, and among the serious candidates there are more than $m-k$ candidates worse than the unique front runner.

\section{$5 k$-Approval voting: the two-voter case}

In this section we concentrate on the two-voter case and on $k$-approval voting. We consider the following question: which $k$-approval rule is least (or minimally) manipulable, under various assumptions on preference extensions as studied in the preceding sections? Answering this question for more than two voters is combinatorially complex, and will be limited to simulation results in Sect. 7; those results also extend to approval voting, including the two-voter case.

We start with a simple theorem, which will be derived from Theorems 4.1, 4.2, and 4.3, but also easily follows directly. It states that $\varphi_{1}$ is strategy-proof, i.e., not manipulable at any profile $p$.

Theorem 5.1 Let $n=2$. Then the 1-approval rule $\varphi_{1}$ is strategy-proof under worst, best, and stochastic dominance comparison.

Proof Let $p=(p(1), p(2))$ be a preference profile and let $k=1$. Note that (b) in Theorem 4.1 is always satisfied: this shows strategy-proofness under worst comparison. In Theorem 4.2, (a) reduces to $\beta(p(1))=\beta(p(2))$ and (b) to $\beta(p(1)) \neq \beta(p(2))$ : this shows strategy-proofness under best comparison. Finally, in Theorem 4.3, (b) reduces to $\beta(p(1)) \neq \beta(p(2))$ and (d) to $\beta(p(1))=\beta(p(2))$ : this shows strategy-proofness under stochastic dominance comparison.

This observation might make our quest for minimally manipulable rules futile, were it not the case that the 1-approval rule (i.e., plurality rule) is not unambiguously attractive. As an example, consider the case where voter 1 has preference $p(1): x z \ldots y$ and voter 2 has preference $p(2): y z \ldots x$ (notations obvious). Then $\varphi_{1}(p)=\{x, y\}$ but $\varphi_{2}(p)=\{z\}$. So it seems that $\varphi_{2}$ offers a better compromise in this case than $\varphi_{1}$. 
Moreover, for more than two voters and apart from a few particular cases, Theorem 5.1 no longer holds.

We consider the three cases (worst, best, and stochastic dominance comparison) separately, in the next three subsections. In a fourth subsection, we consider two further preference extensions-lexicographic worst and best comparison-for the two-voter case.

\subsection{Worst comparison for two voters}

The non-manipulable profiles for two voters under worst comparison are easily described using Theorem 4.1.

Corollary 5.2 Let $n=2$ and $2 \leq k<m$. Let $p \in P$ and consider worst comparison.

(a) If $k \leq(m+1) / 2$, then $\varphi_{k}$ is not manipulable at $p$ if and only if $\left|\varphi_{k}(p)\right|=1$, or equivalently,

$$
\left|\beta_{k}(p(1)) \cap \beta_{k}(p(2))\right|=1 .
$$

(b) If $k>(m+1) / 2$, then $\varphi_{k}$ is not manipulable at $p$ if and only if $\left|\varphi_{k}(p)\right|=2 k-m$, or equivalently,

$$
\left|\beta_{k}(p(1)) \cap \beta_{k}(p(2))\right|=2 k-m .
$$

Proof Case (b) in Theorem 4.1 does not apply. If case (a) in Theorem 4.1 applies then we have $\left|\beta_{k}(p(1)) \cap \beta_{k}(p(2))\right|=1$ (or, equivalently, $\left|\varphi_{k}(p)\right|=1$ ), but this is possible if and only if $k \leq(m+1) / 2$. If case (c) in Theorem 4.1 applies then we have $\left|\beta_{k}(p(1)) \cap \beta_{k}(p(2))\right|=2 k-m$ (or, equivalently, $\left|\varphi_{k}(p)\right|=2 k-m$ ), but this is possible if and only if $k \geq(m+1) / 2$; but for $k=(m+1) / 2$ we have $2 k-m=1$, so that we are back in case (a).

Denote by $\eta(m, k)$ the number of profiles (for two voters) at which $\varphi_{k}$ is not manipulable. By straightforward counting we obtain the following result for the number of manipulable profiles for two voters under worst comparison.

Theorem 5.3 Let $n=2$ and $2 \leq k<m$. Consider worst comparison. Then

$$
\eta(m, k)=\left\{\begin{array}{l}
m ! k\left(\begin{array}{c}
m-k \\
k-1
\end{array}\right) k !(m-k) ! \text { if } k \leq(m+1) / 2 \\
m !\left(\begin{array}{c}
k \\
2 k-m
\end{array}\right) k !(m-k) ! \text { if } k>(m+1) / 2
\end{array}\right.
$$

From this theorem we derive the following corollary (see the Appendix for a proof), which states some facts about $k$ as far as non-manipulability is concerned.

Corollary 5.4 (a) $\eta(m, k)$ increases in $k$ between 2 and an integer $k^{*}$, which is close $^{11}$ to $\sqrt{m}$, and decreases between $k^{*}$ and $\frac{1}{2}(m-1)$.

11 The exact meaning of $k^{*}$ being close to $\sqrt{m}$ in part (a) is explained in the proof. 
(b) $\eta(m, k)$ increases between $\frac{1}{2}(m-1)$ and $(m-1)$.

(c) The $(m-1)$-approval scoring rule is second best since $\eta(m,(m-1))>\eta(m, k)$ for all $m-1>k \geq 2$.

The first-best value of $k$ is $k=1$ (Theorem 5.1), but $\varphi_{1}$ has the drawback that it does not give much opportunity for compromises. Among other values of $k$, the value $k=m-1$ is best. We might, however, prefer to have $k \leq(m+1) / 2$, for the following reason. Call $\varphi_{k}$ citizen-sovereign if for every candidate $x \in A$ there is a profile $p \in P$ with $\varphi_{k}(p)=\{x\}$. It is not difficult to see that $\varphi_{k}$ is citizen-sovereign for any number of voters $n \geq 2$ if $k \leq(m+1) / 2$. For $n=2$ and $k>(m+1) / 2$, however, $\varphi_{k}$ is not citizen-sovereign. Hence, if we restrict ourselves to citizen-sovereign rules with $k \geq 2$, then the best rule is $\varphi_{k^{*}}$, where $k^{*}$ is close to $\sqrt{m}$.

\subsection{Best comparison for two voters}

The non-manipulable profiles for two voters under best comparison can be derived from Theorem 4.2.

Corollary 5.5 Let $n=2$ and $2 \leq k<m$. Let $p \in P$ and consider best comparison.

(a) If $k \leq m / 2$ then $\varphi_{k}$ is not manipulable at $p \in P$ if and only if either

$$
\beta(p(1)) \in \beta_{k}(p(2)) \text { and } \beta(p(2)) \in \beta_{k}(p(1))
$$

or

$$
\beta_{k}(p(1)) \cap \beta_{k}(p(2))=\emptyset .
$$

(b) If $k>m / 2$ then $\varphi_{k}$ is not manipulable at any $p \in P$.

Proof If $k>m / 2$ then case (c) in Theorem 4.2 applies to all $p \in P$, and if $k \leq m / 2$ then case (c) applies to no $p \in P$. This implies part (b) of the corollary, and it also implies that for $k \leq m / 2$ we only have to consider cases (a) and (b) in Theorem 4.2. It is easily seen that these cases result in the two cases in part (a) of the corollary.

The number of non-manipulable profiles $\eta(m, k)$ if $k \leq m / 2$ is computed in the following theorem.

Theorem 5.6 Let $n=2$ and $2 \leq k \leq m / 2$. Consider best comparison. Then

$$
\eta(m, k)=m !(m-2) !(k-1)^{2}+m !(m-1) !+m ![(m-k) !]^{2} /(m-2 k) ! .
$$

Proof The first case in (a) in Corollary 5.5 with $\beta(p(1)) \neq \beta(p(2))$ results in

$$
m !(k-1)\left(\begin{array}{c}
m-2 \\
k-2
\end{array}\right)(k-1) !(m-k) !
$$

different non-manipulable profiles. This yields the first term of $\eta(m, k)$ in the theorem. If $\beta(p(1))=\beta(p(2))$ then this number is simply equal to $m$ ! $(m-1)$ !, which yields the second term. The second case in (a) in Corollary 5.5 results in 


$$
m !\left(\begin{array}{c}
m-k \\
k
\end{array}\right) k !(m-k) !
$$

different non-manipulable profiles, which simplifies to the third term for $\eta(m, k)$ in the theorem.

If we require $k \neq 1$ and citizen-sovereignty, i.e., $k \leq m / 2$, then the optimal value of $k$ with respect to non-manipulability, i.e., the value of $k$ that maximizes $\eta(m, k)$, is equal to 2 .

To see this, note that by Theorem 5.6 and some elementary calculations we have for $2<k \leq \frac{m}{2}$ :

$$
\begin{aligned}
& \eta(m, 2)>\eta(m, k)
\end{aligned}
$$

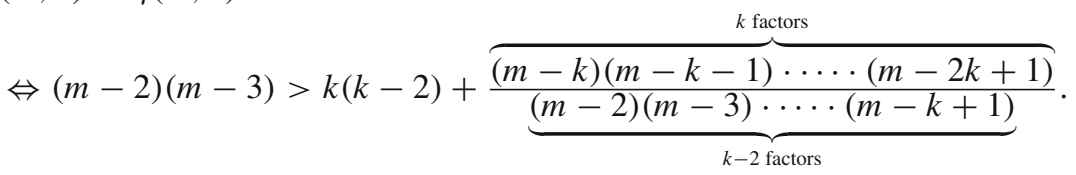

Since $k>2$ it is therefore sufficient to prove that

$$
(m-2)(m-3)>k(k-2)+(m-2 k+2)(m-2 k+1) .
$$

This simplifies to $(4 k-8) m>5 k^{2}-8 k-4$. Since $m \geq 2 k$, it is sufficient to show that $3 k^{2}-8 k+4>0$, which indeed holds for $k>2$.

\subsection{Stochastic dominance comparison for two voters}

The non-manipulable profiles for two voters under stochastic dominance comparison can be derived from Theorem 4.3.

Corollary 5.7 Let $n=2$ and $2 \leq k<m$. Let $p \in P$ and consider stochastic dominance comparison. Then $\varphi_{k}$ is not manipulable at $p$ if and only if at least one of the following holds.

(a) $\beta_{k}(p(1)) \cap \beta_{k}(p(2))=\emptyset$.

(b) $\left|\beta_{k}(p(1)) \cap \beta_{k}(p(2))\right|=1$.

(c) $\beta_{k}(p(1)) \cap \beta_{k}(p(2)) \neq \varnothing$ and $\left[A \backslash \beta_{k}(p(1))\right] \cap\left[A \backslash \beta_{k}(p(2))\right]=\emptyset$.

Proof For $n=2$ and $k \geq 2$ cases (d) and (e) in Theorem 4.3 are not possible. Case (c) in Theorem 4.3 reduces to case (b) above, and case (a) in Theorem 4.3 reduces to case (c) above. Finally, case (b) in the theorem reduces to case (a) above.

From this description we can again derive the number of manipulable profiles $\eta(m, k)$.

Theorem 5.8 Let $n=2, k \geq 2$, and consider stochastic dominance comparison.

(a) If $k \leq m / 2$ then

$$
\eta(m, k)=m ![(m-k) !]^{2} /(m-2 k) !+m ! k^{2}[(m-k) !]^{2} /(m-2 k+1) ! .
$$


(b) If $k>m / 2$ then

$$
\eta(m, k)=m ![k !]^{2} /(2 k-m) !
$$

Proof If $k \leq m / 2$ then (c) in Corollary 5.7 is not possible, and cases (a) and (b) in the corollary are mutually exclusive. In case (a) of Corollary 5.7 there are

$$
m !\left(\begin{array}{c}
m-k \\
k
\end{array}\right) k !(m-k) !
$$

non-manipulable profiles, resulting in the first term for $\eta(m, k)$, and in case (b) of the corollary there are

$$
m ! k\left(\begin{array}{c}
m-k \\
k-1
\end{array}\right) k !(m-k) !
$$

non-manipulable profiles, resulting in the second term for $\eta(m, k)$.

If $k>m / 2$ then case (a) of Corollary 5.7 is not possible, and (b) is a special case of (c). For the latter case, we just have to count the number of profiles for which $\left[A \backslash \beta_{k}(p(1))\right] \cap\left[A \backslash \beta_{k}(p(2))\right]=\emptyset$, since the other condition is always fulfilled. This number is equal to

$$
m !\left(\begin{array}{c}
k \\
m-k
\end{array}\right) k !(m-k) !
$$

which is equal to $m ![k !]^{2} /(2 k-m) !$.

About the value of $k$ that maximizes $\eta(m, k)$, so the value of $k$ that is optimal with respect to non-manipulability, we can say the following.

1. For $2 \leq k \leq \frac{m}{2}$, the number of non-manipulable profiles decreases with $k$, and thus $k=2$ is optimal.

2. For $\frac{m}{2}<k \leq m-1$, the number of non-manipulable profiles increases with $k$, and thus $k=m-1$ is optimal.

3. $\eta(m, 2)>\eta(m, m-1)$ for $m \geq 4$, so $k=2$ is the overall optimal value between 2 and $m-1$.

To prove these statements, first assume $k \leq \frac{m}{2}$. Then, using Theorem 5.8(a) and simplifying, we derive

$$
\eta(m, k+1)<\eta(m, k) \Leftrightarrow 3 k^{2}-2 k m-1<0,
$$

and it is easily seen that the right hand side holds for all $2 \leq k \leq \frac{m}{2}$. Next, assume $\frac{m}{2}<k \leq m-1$. Then, using Theorem 5.8(b) and simplifying, we derive

$$
\eta(m, k+1)>\eta(m, k) \Leftrightarrow 3 k^{2}+k(4-4 m)+m^{2}-3 m+1<0 .
$$


The roots of the quadratic expression in $k$ at the right hand side are $\frac{2}{3}(m-1) \pm$ $\frac{1}{3} \sqrt{m^{2}+m+1}$; the smaller root is smaller than $\frac{m}{2}$, whereas the larger root is larger than $m-1$. Thus, the right hand side holds for all $\frac{m}{2}<k \leq m-1$. Finally, by Theorem 5.8 again,

$$
\eta(m, 2)>\eta(m, m-1) \Leftrightarrow m>3,
$$

so that $k=2$ is the overall optimal value of $k$ for $2 \leq k \leq m-1$.

\subsection{Lexicographic worst and best comparison}

In this section we briefly consider a natural extension of worst and best comparison, namely lexicographic worst and best comparison. These preference extensions to sets are given by the following recursive definition. For two subsets $B$ and $C$ of candidates, we say that $B$ is (weakly) preferred to $C$ under lexicographic worst comparison by voter $i$ with preference $p(i)$ if

1. $C=\emptyset$, or

2. $B$ and $C$ are non-empty and $\left(\omega\left(p(i)_{\mid B}\right), \omega\left(p(i)_{\mid C}\right)\right) \in p(i)$, or

3. $\omega\left(p(i)_{\mid B}\right)=\omega\left(p(i)_{\mid C}\right)=: w$ and $B \backslash\{w\}$ is preferred to $C \backslash\{w\}$ under lexicographic worst comparison by voter $i$ with preference $p(i)$.

The definition for lexicographic best comparison is obtained by replacing the worst candidate by the best candidate, i.e., by replacing $\omega(\cdot)$ by $\beta(\cdot)$. Thus, under lexicographic worst comparison a voter first considers the worst elements of $B$ and $C$. If these are different, then he prefers the set with the better worst element. Otherwise, the voter considers the second worst elements. If these are different, then he prefers the set with the better second worst element. Otherwise, he considers the third worst elements, etc. Similarly for lexicographic best comparison.

Complete characterizations of the non-manipulable profiles for both lexicographic worst and lexicographic best comparison for arbitrary $n$ can be given but are rather technical (even more so than for stochastic dominance comparison) and therefore not included.

Note that any profile that is manipulable under worst [best] comparison is also manipulable under lexicographic worst [best] comparison. Hence, the set of nonmanipulable profiles under lexicographic worst [best] comparison is always a subset of the set of non-manipulable profiles under worst [best] comparison. It is not very difficult to check (we omit the proof for the sake of briefness) that all the profiles listed in Corollary 5.2, that is, all two-voter profiles that are non-manipulable under worst comparison, are also non-manipulable under lexicographic worst comparison, so that in this case considering lexicographic worst comparison instead of just worst comparison does not make any difference. The non-manipulable profiles coincide, and the optimal value of $k$ as far as non-manipulability is concerned, is the same as in Sect. 5.1.

For two voters and lexicographic best comparison the situation is different and the set of non-manipulable profiles is a strict subset of the set of non-manipulable profiles 
under best comparison, that is, the set of profiles described in Corollary 5.5. To be precise, we have the following result, which can be derived from Corollary 5.5 (the proof is again left to the reader).

Corollary 5.9 Let $n=2$ and $2 \leq k<m$. Let $p \in P$ and consider lexicographic best comparison.

(a) If $k \leq m / 2$ then $\varphi_{k}$ is not manipulable at $p \in P$ if and only if either

$$
\{\beta(p(1))\}=\{\beta(p(2))\}=\beta_{k}(p(1)) \cap \beta_{k}(p(1))
$$

or

$$
\beta_{k}(p(1)) \cap \beta_{k}(p(2))=\varnothing .
$$

(b) If $k>m / 2$ then $\varphi_{k}$ is not manipulable at any $p \in P$.

In this case, the total number of non-manipulable profiles for $2 \leq k \leq m / 2$ is equal to

$$
\eta(m, k)=\frac{m ![(m-k) !]^{2}(m-2 k+2)}{(m-2 k+1) !}
$$

and this number is decreasing in $k$, so that $k=2$ is the value of $k$ that minimizes manipulability subject to $2 \leq k \leq m / 2$, just as in the best comparison case. The proofs of these facts are somewhat simpler than for the best comparison case. For the sake of briefness we omit them.

\section{An asymptotic result}

We start with defining the class of all scoring rules. A (normalized) scoring vector is a vector $s=\left(s_{1}, s_{2}, \ldots, s_{m}\right) \in \mathbb{R}^{m}$ with $1=s_{1} \geq s_{2} \geq \ldots \geq s_{m}=0$. For a preference $\pi \in P$ and a candidate $x \in A$ let $t(\pi, x)$ denote the rank of $x$ in the preference $\pi$, i.e., $t(\pi, x)=k$ where $k=1$ if $\{x\}=\beta(\pi)$ and $\{x\}=\beta_{k}(\pi) \backslash \beta_{k-1}(\pi)$ otherwise.

For a scoring vector $s$, a profile $p \in P^{N}$, and a candidate $x \in A$, we denote by the s-score

$$
\operatorname{score}_{s}(x, p)=\sum_{i \in N} s_{t(p(i), x)}
$$

the total score that $x$ obtains under profile $p$ and score vector $s$. The scoring rule with scoring vector $s$ is defined by

$$
\varphi_{s}(p)=\left\{x \in A \mid \operatorname{score}_{s}(x, p) \geq \operatorname{score}_{s}(y, p) \text { for all } y \in A\right\}, \quad p \in P^{N} .
$$

Clearly, a $k$-approval rule is a scoring rule with scoring vector $s$ such that $s_{1}=\cdots=$ $s_{k}=1$ and $s_{k+1}=\cdots=s_{m}=0$. 
Now, in what follows, we fix the number of candidates $m$ and let the number of voters go to infinity. We will show, formally, that then any two scoring rules lead to the same expected values of the highest score, second highest score, and so on, up to a multiplicative constant proportional to the standard deviations of the scoring vectors: the higher this standard deviation the larger the differences between the expected scores. Since the standard deviation is maximal for $k$-approval rules with $k$ around $m / 2$, we can conclude by the law of large numbers that the proportion of manipulable profiles is smallest for this rule. ${ }^{12}$

In order to derive the announced result, assume that voter preferences are drawn from the uniform distribution over $P$-that is, according to 'impartial culture'. Let $Y=\left(Y_{\pi}\right)_{\pi \in P}$ denote the random vector giving the numbers of voters for each preference (so $\sum_{\pi \in P} Y_{\pi}=n$ ). Then $Y$ has a multinomial distribution with mean $(n / m !) \mathbf{1}$, where 1 is a vector with all entries equal to 1 . Write $A=\left\{x_{1}, \ldots, x_{m}\right\}$, then for a scoring vector $s$ the random vector $Y$ gives rise to a random vector of scores $X^{s}=$ $\left(X_{1}^{s}, \ldots, X_{m}^{s}\right)$ where $X_{j}^{s}=\sum_{\pi \in P} Y_{\pi} s_{t\left(\pi, x_{j}\right)}$ for $j=1, \ldots, m$. Let

$$
\sigma(s)=\sqrt{\frac{s_{1}^{2}+\cdots+s_{m}^{2}}{m}-\bar{s}^{2}}
$$

denote the standard deviation of the scoring vector $s$, where $\bar{s}=\left(s_{1}+\cdots+s_{m}\right) / m$ is the mean of $s$. Proposition 2 in Pritchard and Wilson (2009) asserts that $\left(X^{s}-\right.$ $n \bar{s} \mathbf{1}) / \sqrt{n}$ converges in distribution to $Z^{s}:=\sigma(s)\left(\frac{m}{m-1}\right)^{1 / 2}(Z-\bar{Z} \mathbf{1})$, where $Z=$ $\left(Z_{1}, \ldots, Z_{m}\right)$ is a vector of independent standard normal random variables and $\bar{Z}=$ $(1 / m) \sum_{j=1}^{m} Z_{j}$. In words, this means that the limit distributions of the vectors of normalized random variables $X^{s}$ differ only in a multiplicative constant, namely the standard deviation $\sigma(s)$. This implies $Z^{s}=\left(\sigma(s) / \sigma\left(s^{\prime}\right)\right) Z^{s^{\prime}}$ for any two scoring vectors $s$ and $s^{\prime}$. In particular, this also holds for the associated order statistics $\left(Z_{(1)}^{s}, \ldots, Z_{(m)}^{s}\right)$ and $\left(Z_{(1)}^{s^{\prime}}, \ldots, Z_{(m)}^{s^{\prime}}\right)$, with $Z_{(1)}^{s}$ and $Z_{(1)}^{s^{\prime}}$ being the (limit) distributions of the highest scores. As a consequence we obtain the following proposition.

Proposition 6.1 For all scoring vectors $s$ and $s^{\prime}$,

$$
E\left[Z_{(j)}^{s}\right]-E\left[Z_{(j+1)}^{s}\right]=\left(\sigma(s) / \sigma\left(s^{\prime}\right)\right)\left(E\left[Z_{(j)}^{s^{\prime}}\right]-E\left[Z_{(j+1)}^{s^{\prime}}\right]\right)
$$

for all $j=1, \ldots, m-1$, where $E$ denotes the expectation operator.

Proposition 6.1 implies that the difference in expected value between any two consecutive scores is largest for rules based on scoring vectors with maximal standard deviation. Since the vectors of random variables $Z^{s}$ have the same distributions up to these standard deviations of the score vectors, and taking into account that the probability of all candidates having distinct scores converges to 1 if the number of voters

12 We thank Eric Beutner (Maastricht University) for helpful discussions on this topic. 
goes to infinity, ${ }^{13}$ we have by the law of large numbers that scoring rules $\varphi_{s}$ with maximal standard deviation $\sigma(s)$ have the smallest proportion of manipulable profiles. The following result, of which for completeness a proof is given in the Appendix, then implies that the $k$-approval rule with $k$ around $m / 2$ is least manipulable if the number of voters becomes large.

Proposition 6.2 Among all scoring vectors $s, \sigma(s)$ is maximal if and only if $s_{1}=$ $\cdots=s_{k}=1$ and $s_{k+1}=\cdots=s_{m}=0$, where $k=m / 2$ if $m$ is even and $k \in$ $\{(m-1) / 2,(m+1) / 2\}$ if $m$ is odd.

For ease of reference we formulate the main result of this section as a corollary.

Corollary 6.3 Let $k^{*}$ denote the value(s) of $k$ in Proposition 6.2 and let s be an arbitrary scoring vector unequal to the scoring vector associated with $k^{*}$. Then for $n$ sufficiently large the proportion of manipulable profiles under $\varphi_{k^{*}}$ is smaller than the proportion of manipulable profiles under $\varphi_{s}$.

This asymptotic result should be taken with some care, since the probability of being able to manipulate becomes very small if the number of voters grows, and so we are comparing small numbers. On the other hand there is some evidence that already for a relatively small number of voters the $k$-approval rule with $k$ close to $m / 2$ performs best, at least among the $k$-approval rules. See Table 3 in the next section.

\section{Some simulation results}

Since general comparisons between the approval rule and $k$-approval rules are complex and hard to obtain, we present here some results of simulations. ${ }^{14}$

Table 1 gives the approximate percentages of non-manipulable profiles for the approval rule with 3-10 candidates and 2, 3, 6, and 10 voters, based on 1,00,000 trials. While the number of trials is relatively low, we nevertheless think that the numbers in the table give reliable impressions.

Some conclusions can be drawn from this table. Clearly, the possibility of manipulation increases with the number of candidates. For more than two voters manipulability also increases from worst comparison to best comparison and from best comparison to stochastic dominance comparison. This is not entirely intuitive at first glance. One might expect that many profiles that are manipulable under stochastic comparison are also manipulable under worst and best comparison, since in order to improve under stochastic comparison a necessary condition is that the worst and best candidates of a set should not decrease in preference. Thus, to explain the results in Table 1, it seems to be the case that manipulation under stochastic comparison is often performed by improving intermediate candidates. Moreover, apparently this kind of manipulation

\footnotetext{
13 This is Proposition 3 in Pritchard and Wilson (2009).

14 We thank Bram Driesen for doing these simulations (with Matlab). They are based on constructing random profiles by randomly drawing strict preferences and checking for non-manipulability using the characterizations in Sects. 3 and 4. For approval voting, a profile is constructed by randomly drawing a strict preference $w(i)$ and a 'cut-off' number $k(i)$ for each voter $i$.
} 
Table 1 Approximate percentages of non-manipulable preference profiles for the approval rule, based on $1,00,000$ trials

\begin{tabular}{|c|c|c|c|c|c|c|c|c|c|}
\hline & $m$ & 3 & 4 & 5 & 6 & 7 & 8 & 9 & 10 \\
\hline \multirow[t]{3}{*}{$n=2$} & Worst comp & 56 & 45 & 38 & 33 & 30 & 27 & 25 & 23 \\
\hline & Best comp & 65 & 59 & 55 & 52 & 49 & 48 & 46 & 45 \\
\hline & Stoch comp & 41 & 37 & 34 & 31 & 30 & 28 & 27 & 25 \\
\hline \multirow[t]{3}{*}{$n=3$} & Worst comp & 57 & 49 & 44 & 41 & 38 & 36 & 34 & 32 \\
\hline & Best comp & 56 & 47 & 41 & 38 & 34 & 32 & 30 & 28 \\
\hline & Stoch comp & 25 & 20 & 17 & 16 & 15 & 14 & 14 & 13 \\
\hline \multirow[t]{3}{*}{$n=6$} & Worst comp & 67 & 61 & 57 & 54 & 52 & 50 & 48 & 47 \\
\hline & Best comp & 51 & 40 & 33 & 29 & 25 & 22 & 20 & 18 \\
\hline & Stoch comp & 28 & 20 & 16 & 13 & 11 & 10 & 09 & 08 \\
\hline \multirow[t]{3}{*}{$n=10$} & Worst comp & 73 & 69 & 65 & 62 & 60 & 59 & 57 & 56 \\
\hline & Best comp & 54 & 43 & 37 & 32 & 28 & 25 & 23 & 21 \\
\hline & Stoch comp & 35 & 27 & 22 & 19 & 16 & 15 & 13 & 12 \\
\hline
\end{tabular}

Table 2 Approximate percentages of non-manipulable preference profiles for $k$-approval rules and the approval rule, $m=6$, based on 1,00,000 trials

\begin{tabular}{llrllrrl}
\hline$(m=6)$ & $k$ & 1 & 2 & 3 & \multicolumn{1}{l}{4} & \multicolumn{1}{l}{5} & Approval rule \\
\hline$n=2$ & Worst comp & 100 & 54 & 45 & 40 & 83 & 33 \\
& Best comp & 100 & 60 & 35 & 100 & 100 & 52 \\
& Stoch comp & 100 & 93 & 50 & 40 & 83 & 31 \\
$n=3$ & Worst comp & 44 & 57 & 61 & 32 & 56 & 41 \\
& Best comp & 100 & 63 & 51 & 48 & 100 & 38 \\
& Stoch comp & 100 & 30 & 54 & 35 & 56 & 16 \\
$n=6$ & Worst comp & 60 & 64 & 60 & 51 & 25 & 54 \\
& Best comp & 50 & 59 & 50 & 46 & 78 & 29 \\
& Stoch comp & 34 & 41 & 24 & 16 & 16 & 13 \\
$n=10$ & Worst comp & 69 & 70 & 68 & 64 & 56 & 62 \\
& Best comp & 45 & 56 & 52 & 40 & 51 & 32 \\
& Stoch comp & 29 & 36 & 31 & 27 & 07 & 19 \\
\hline
\end{tabular}

The percentages equal to 100 are exact and reflect strategy-proofness in the involved cases

can often lead to comparable sets, in spite of the fact that stochastic dominance preference is not complete.

As a final comment on Table 1, under worst comparison manipulability seems to decrease with the number of voters, but under the other two preference extensions manipulability first seems to increase and then to decrease again.

In Table 2 we present the results for $k$-approval rules for six candidates; 2, 3, 6, or 10 voters; and again based on 1,00,000 trials. For comparison the corresponding results for the approval rule from Table 1 are copied in Table 2. 
Table 3 Percentages of non-manipulable preference profiles for $k$-approval rules and the approval rule, $m=10, n=25$, based on $1,000,000$ trials

\begin{tabular}{llllllll}
\hline$m=10$ & $k$ & 1 & 3 & 5 & 7 & 9 & Approval rule \\
\hline$n=25$ & Worst comp & 67.1 & 74.4 & 74.5 & 70.2 & 48.8 & 69.5 \\
& Best comp & 33.5 & 49.9 & 50.2 & 40.9 & 54.8 & 37.6 \\
& Stoch comp & 26.6 & 37.6 & 37.8 & 29.7 & 03.7 & 29.1 \\
\hline
\end{tabular}

Again we see that for relatively high numbers of voters manipulability seems to increase from worst to best and from best to stochastic dominance comparison. Further, for more than two voters the approval rule is outperformed by (at least) the 3-approval rule as far as non-manipulability is concerned.

The final simulation results we present are collected in Table 3. This table gives the percentages of non-manipulable preference profiles for $k$-approval rules with $k$ odd and the approval rule for 10 candidates, 25 voters, based on 1,000,000 trials. We give more accurate numbers than in the other tables since some differences are very small. A prudent observation is that the 5-approval rule performs best with respect to (non-)manipulability among the odd values of $k$ (except for the case $k=9$ and best comparison)_-in line with the asymptotic result in Sect. 6. Also, it performs better than the approval rule.

\section{Concluding remarks}

The main conclusions from this paper can be summarized as follows. For the case of two voters we have established which $k$-approval voting rule is optimal in terms of minimizing the number of manipulable profiles. The optimal $k$ depends on the particular preference extension. Excluding $k=1$ (plurality voting, strategy-proof but otherwise less attractive, as argued) and $k>(m+1) / 2$ (not citizen-sovereign), for worst comparison and lexicographic worst comparison a value of $k$ close to $\sqrt{m}$ is optimal; for stochastic dominance, best comparison and lexicographic best comparison $k=2$ is optimal. On the other extreme, if the number of voters becomes large then the $k$-approval rule with $k$ close to half of the number of candidates is even best among all scoring rules. Simulations show that this may also be the case if the number of voters is relatively large. With only a few exceptions, simulations show that approval voting is more manipulable (percentage-wise) than $k$-approval voting.

Of course, these conclusions should be interpreted with care. They are based on the impartial culture assumption, assuming that all preference profiles are equally likely, and they do not say anything about how difficult it is to manipulate or how much gain can be obtained from manipulation (cf. Campbell and Kelly 2009). Moreover, while $k$-approval tends to be less manipulable than approval voting, manipulation under approval voting still maintains sincerity, which is not the case for $k$-approval voting.

Even though our measure of non-manipulability reflects 'impartial culture' (each preference profile is regarded as equally likely) the characterizations of the sets of 
non-manipulable profiles derived in this paper, however, are also needed when considering 'partial culture'.

Acknowledgments We thank participants of conferences in Tilburg (May 2009) and Caen (June 2009) for helpful discussions on this subject. We also thank Vincent Merlin and two referees for their informative comments.

Open Access This article is distributed under the terms of the Creative Commons Attribution Noncommercial License which permits any noncommercial use, distribution, and reproduction in any medium, provided the original author(s) and source are credited.

\section{Appendix: Remaining proofs}

Proof of Theorem 3.5 For the if-part, let $s$ be an $i$-deviation of $r$, with $s(i)=(q(i)$, $\ell(i))$.

In case (a), $\varphi(r)=\varphi\left(r_{-i}\right) \cup\left[\varphi^{-}\left(r_{-i}\right) \cap \beta_{k(i)}(w(i))\right]$. If $\varphi\left(r_{-i}\right) \cap \beta_{\ell(i)}(q(i))=\varnothing$ then $\varphi(s)=\varphi\left(r_{-i}\right) \cup\left[\varphi^{-}\left(r_{-i}\right) \cap \beta_{\ell(i)}(q(i))\right]$ but this is not preferred to $\varphi(r)$ according to $\succeq_{w(i)}$. If $\varphi\left(r_{-i}\right) \cap \beta_{\ell(i)}(q(i)) \neq \varnothing$ then $\varphi(s)=\varphi\left(r_{-i}\right) \cap \beta_{\ell(i)}(q(i))$ which again is not preferred to $\varphi(r)$ according to $\succeq_{w(i)}$.

In case (b), $\varphi(r)=\varphi\left(r_{-i}\right) \cap \beta_{k(i)}(w(i))$ and $\{x\} \sim_{w(i)}\{y\}$ for all $x, y \in \varphi(r)$. If $\varphi(s) \subseteq \varphi(r)$ then $\varphi(s)$ is not preferred to $\varphi(r)$. Otherwise, there are $z \in \varphi(s) \cap \varphi\left(r_{-i}\right)$ with $(x, z) \in w(i)$ for all $x \in \varphi(r)$. In that case too, $\varphi(s)$ is not preferred to $\varphi(r)$ according to $\succeq_{w(i)}$.

For case (c), note that $\varphi(r) \subseteq \varphi\left(r_{-i}\right)$ and thus, $\{x\} \sim_{w(i)}\{y\}$ for all $x, y \in \varphi(r)$. Also, $\{x\} \succeq_{w(i)}\{y\}$ for all $x \in \varphi\left(r_{-i}\right)$ and $y \in \varphi^{-}\left(r_{-i}\right)$. So $\varphi(s) \nsucc_{w(i)} \varphi(r)$.

For the only-if part, suppose that there is a voter $i \in N$ such that none of the three cases (a)-(c) holds. It is sufficient to prove that $\varphi(r)$ is manipulable at profile $r$ by voter $i$. By taking negations it follows that none of (a)-(c) holding is equivalent to all of the following three statements holding for $r$ and $i$.

1. (i) $\varphi\left(r_{-i}\right) \nsubseteq A \backslash \beta_{k(i)}(w(i))$ or (ii) $\varphi^{-}\left(r_{-i}\right) \cap \beta_{k(i)}(w(i))=\emptyset$.

2. (i) $\varphi\left(r_{-i}\right) \cap \beta_{k(i)}(w(i))=\emptyset$ or (ii) for some $x, y \in \varphi\left(r_{-i}\right) \cap \beta_{k(i)}(w(i))$ $\{x\} \succ_{w(i)}\{y\}$ or (iii) $A \backslash \beta_{k(i)}(w(i)) \cap \varphi\left(r_{-i}\right)=\emptyset$.

3. (i) $\{x\} \succ_{w(i)}\{y\}$ for some $x, y \in \varphi\left(r_{-i}\right)$ or (ii) there are $x \in \varphi^{-}\left(r_{-i}\right)$ such that $\{x\} \succ_{w(i)} \varphi\left(r_{-i}\right)$.

Requiring (1)-(3) to hold implies twelve cases to consider. However, 1(i) and 2(i) are contradictory, so that ten cases are left. Now cases 1(i) \& 2(ii) \& 3(i); 1(i) \& 2(ii) \& 3(ii); 1(i) \& 2(iii) \& 3(i); 1(ii) \& 2(ii) \& 3(i); 1(ii) \& 2(ii) \& 3(ii); and 1(ii) \& 2(iii) $\& 3($ i) are covered by case I below. Case 1 (ii) \& 2(i) \& 3(i) is covered by case II. Cases 1 (i) \& 2(iii) \& 3(ii) and 1(ii) \& 2(iii) \& 3(ii) are covered by case III. Lastly, case 1(ii) \& 2(i) \& 3(ii) is covered by case IV.

I. $\varphi\left(r_{-i}\right) \cap \beta_{k(i)}(w(i)) \neq \emptyset$ and there exist $x, y \in \varphi\left(r_{-i}\right) \cap \beta_{k(i)}(w(i))$ such that $\{x\} \succ_{w(i)}\{y\}$.

II. $\varphi\left(r_{-i}\right) \subseteq\left[A \backslash \beta_{k(i)}(w(i))\right], \varphi^{-}\left(r_{-i}\right) \subseteq\left[A \backslash \beta_{k(i)}(w(i))\right]$ and there exist $x, y \in$ $\varphi\left(r_{-i}\right)$ such that $\{x\} \succ_{w(i)}\{y\}$.

III. $\varphi\left(r_{-i}\right) \subseteq \beta_{k(i)}(w(i))$ and there exist $x \in \varphi^{-}\left(r_{-i}\right)$ such that $\{x\} \succ_{w(i)}\{y\}$ for all $y \in \varphi\left(r_{-i}\right)$. 
IV. $\varphi\left(r_{-i}\right) \subseteq\left[A \backslash \beta_{k(i)}(w(i))\right], \varphi^{-}\left(r_{-i}\right) \subseteq\left[A \backslash \beta_{k(i)}(w(i))\right]$ and there exist $x \in$ $\varphi^{-}\left(r_{-i}\right)$ such that $\{x\} \succ_{w(i)}\{y\}$ for all $y \in \varphi\left(r_{-i}\right)$.

In cases I and II, take $b \in \beta\left(w(i)_{\mid \varphi\left(r_{-i}\right)}\right)$ and consider the report $s(i)=\left(w^{\prime}(i), 1\right)$ of voter $i$ such that $\beta\left(w^{\prime}(i)\right)=\{b\}$. Then by (1), $\varphi(s)=\{b\}$. In case I, $\varphi(r)=$ $\varphi\left(r_{-i}\right) \cap \beta_{k(i)}(w(i))$, so by the second condition in I, $\varphi(s)=\{b\} \succ_{w(i)} \varphi(r)$. In case II, $\varphi(r)=\varphi\left(r_{-i}\right)$, and by the last condition in case II, again $\varphi(s)=\{b\} \succ_{w(i)} \varphi(r)$.

In both cases III and IV, $\varphi(r)=\varphi\left(r_{-i}\right)$. Take $x \in \varphi^{-}\left(r_{-i}\right)$ such that $\{x\} \succ_{w(i)}\{y\}$ for all $y \in \varphi\left(r_{-i}\right)$. Consider the report $s(i)=\left(w^{\prime}(i), 1\right)$ of voter $i$ such that $\beta\left(w^{\prime}(i)\right)=$ $\{x\}$. Then by (1), $\varphi(s)=\{x\} \cup \varphi\left(r_{-i}\right)$. This implies $\varphi(s)=\{b\} \succ_{w(i)} \varphi(r)$.

Proof of Corollary 3.7 Let $r \in R_{d}^{N}, r(i)=(w(i), k(i))$ for all $i \in N$.

Suppose that for some $j \in N$ statement (b) in Theorem 3.1 does not hold. Then there is $x \in \varphi\left(r_{-j}\right)$ with $x \in \beta_{k(j)}(w(j))$, and, clearly, $\{x\} \sim_{w(j)}\{y\}$ for all $x, y \in$ $\varphi\left(r_{-j}\right) \cap \beta_{k(j)}(w(j))$. Hence, (a) holds for $j$. Thus, $\varphi$ is strategy-proof under worst comparison.

Next, suppose that (a) in Theorem 3.3 does not hold for some $i \in N$. Then there is a $y \in \varphi^{-}\left(r_{-i}\right)$ with $(y, x) \in w(i)$ and $(x, y) \notin w(i)$ for all $x \in \varphi\left(r_{-i}\right)$. This implies that (b) holds for $i$. Thus, $\varphi$ is strategy-proof under best comparison.

Finally, suppose (c) in Theorem 3.5 does not hold for some $i \in N$. There are two cases. If the first statement in (c) does not hold, then $\varphi\left(r_{-i}\right) \cap \beta_{k(i)}(w(i)) \neq \varnothing$ and $\varphi\left(r_{-i}\right) \cap\left[A \backslash \beta_{k(i)}(w(i))\right] \neq \emptyset$, so that (b) holds. If the second statement in (c) does not hold, then there is $y \in \varphi^{-}\left(r_{-i}\right)$ with $\{y\} \succ_{w(i)}\{x\}$ for some $x \in \varphi\left(r_{-i}\right)$. In this case, if $\varphi\left(r_{-i}\right) \subseteq\left[A \backslash \beta_{k(i)}(w(i))\right]$ then (a) holds, and otherwise (b) holds. Thus, $\varphi$ is strategy-proof under stochastic dominance comparison.

Proof of Theorem 4.3 For the if-part, let $q$ be an $i$-deviation of $p$. Note that $\varphi_{k}\left(p_{-i}\right)=$ $\varphi_{k}\left(q_{-i}\right)$ and $\varphi_{k}^{-}\left(p_{-i}\right)=\varphi_{k}^{-}\left(q_{-i}\right)$. Assume that at least one of the cases (a)-(e) holds. We show that voter $i$ cannot manipulate from $p$ to $q$.

In case (a), we have $\varphi_{k}(p)=\varphi_{k}\left(p_{-i}\right) \cap \beta_{k}(p(i))$ and $\varphi_{k}(q)=\varphi_{k}(p)$ or $\varphi_{k}(q) \cap$ $\left[A \backslash \beta_{k}(p(i))\right] \neq \emptyset$. In both cases, it is easy to see that $\varphi_{k}(p) \succeq_{p(i)} \varphi_{k}(q)$.

In case (b), $\varphi_{k}(p)=\varphi_{k}\left(p_{-i}\right) \cup\left[\varphi_{k}^{-}\left(p_{-i}\right) \cap \beta_{k}(p(i))\right]$. If $\beta_{k}(q(i)) \cap \varphi_{k}\left(p_{-i}\right)=\varnothing$ then $\varphi_{k}(q)=\varphi_{k}\left(p_{-i}\right) \cup\left[\varphi_{k}^{-}\left(p_{-i}\right) \cap \beta_{k}(q(i))\right]$ but this is never preferred to $\varphi_{k}(p)$ according to $\succeq_{p(i)}$. If $\beta_{k}(q(i)) \cap \varphi_{k}\left(p_{-i}\right) \neq \emptyset$ then $\varphi_{k}(q)=\beta_{k}(q(i)) \cap \varphi_{k}\left(p_{-i}\right)$, which again is never preferred to $\varphi_{k}(p)$ according to $\succeq_{p(i)}$.

In case (c), (d), and (e), $\varphi_{k}(p)=\{w\}$. If $\varphi_{k}(q) \neq\{w\}$ then $x \in \varphi_{k}(q)$ for some $x \in L(w, p(i)) \backslash\{w\}$. In that case $\varphi_{k}(q)$ is never preferred to $\varphi_{k}(p)$ according to $\succeq p(i)$.

For the only-if part, suppose that there is a voter $i \in N$ such that none of the five cases (a)-(e) holds. It is sufficient to prove that $\varphi_{k}$ is manipulable at profile $p$ by voter $i$.

By taking negations it follows that none of (a)-(e) holding is equivalent to all of the following four statements holding for $p$ and $i$.

I. (i) $A \backslash \beta_{k}(p(i)) \nsubseteq \varphi_{k}\left(p_{-i}\right)$ or (ii) $A \backslash \beta_{k}(p(i))=\varphi_{k}\left(p_{-i}\right)$.

II. (i) $\varphi_{k}\left(p_{-i}\right) \nsubseteq\left[A \backslash \beta_{k}(p(i))\right]$ or (ii) $\varphi_{k}^{-}\left(p_{-i}\right) \cap \beta_{k}(p(i))=\emptyset$. 
III. (i) $\left|\varphi_{k}\left(p_{-i}\right) \cap \beta_{k}(p(i))\right| \geq 2$ or (ii) $\varphi_{k}\left(p_{-i}\right) \cap \beta_{k}(p(i))=\emptyset$ or (iii) $\varphi_{k}\left(p_{-i}\right) \subseteq$ $\beta_{k}(p(i))$.

IV. (i) $\left|\varphi_{k}\left(p_{-i}\right)\right|>1$ or (ii) $\left[\varphi_{k}\left(p_{-i}\right)=\{w\}\right.$ for some $w \in A$ and $\varphi_{k}^{-}\left(p_{-i}\right) \not$ $L(w, p(i))$ and $\left.\left|\varphi_{k}^{-}\left(p_{-i}\right) \cap L(w, p(i))\right| \leq m-k\right]$.

Requiring (I)-(IV) to hold implies 24 cases to consider. However, the following combinations are contradictory: I(ii) and III(i); I(ii) and III(iii); II(i) and III(ii); and III(i) and IV(ii). Moreover, III(iii) and IV(i) together imply III(i), so that we do not have the case with IV(i) and III(iii) separately. This leaves eight cases, which we will consider two at a time.

A. Cases I(i) \& II(i) \& III(i) \& IV(i) and I(i) \& II(ii) \& III(i) \& IV(i). These two cases are covered by the following assumptions: $\left|\varphi_{k}\left(p_{-i}\right) \cap \beta_{k}(p(i))\right| \geq 2$ and $A \backslash \beta_{k}(p(i)) \nsubseteq \varphi_{k}\left(p_{-i}\right)$.

In this case we have $\varphi_{k}(p)=\varphi_{k}\left(p_{-i}\right) \cap \beta_{k}(p(i))$. Let

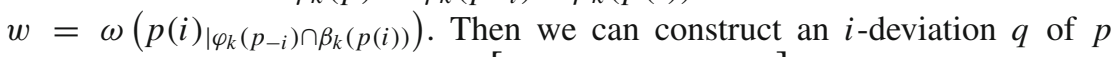
such that $\varphi_{k}\left(p_{-i}\right) \cap \beta_{k}(q(i))=\left[\varphi_{k}\left(p_{-i}\right) \cap \beta_{k}(p(i))\right] \backslash\{w\}$. Then $\varphi_{k}(q)=$ $\left[\varphi_{k}\left(p_{-i}\right) \cap \beta_{k}(p(i))\right] \backslash\{w\}$ and, thus, $\varphi_{k}(q) \succeq_{p(i)} \varphi_{k}(p)$.

B. Cases I(i) \& II(ii) \& III(ii) \& IV(i) and I(ii) \& II(ii) \& III(ii) \& IV(i). These two cases are covered by the following assumptions: $\left|\varphi_{k}\left(p_{-i}\right)\right|>1$ and $\varphi_{k}\left(p_{-i}\right) \cap$ $\beta_{k}(p(i))=\emptyset$ and $\varphi_{k}^{-}\left(p_{-i}\right) \cap \beta_{k}(p(i))=\emptyset$.

In this case we have $\varphi_{k}(p)=\varphi_{k}\left(p_{-i}\right)$. Let $b=\beta\left(p(i)_{\mid \varphi_{k}\left(p_{-i}\right)}\right)$. We can construct an $i$-deviation $q$ of $p$ such that $\varphi_{k}\left(p_{-i}\right) \cap \beta_{k}(q(i))=\{b\}$. Then $\varphi_{k}(q)=$ $\{b\} \succ_{p(i)} \varphi_{k}\left(p_{-i}\right)=\varphi_{k}(p)$.

C. Cases I(i) \& II(ii) \& III(ii) \& IV(ii) and I(ii) \& II(ii) \& III(ii) \& IV(ii). These two cases are covered by the following assumptions: $\left[\varphi_{k}\left(p_{-i}\right)=\{w\}\right.$ for some $w \in A$ and $\varphi_{k}^{-}\left(p_{-i}\right) \nsubseteq L(w, p(i))$ and $\left.\left|\varphi_{k}^{-}\left(p_{-i}\right) \cap L(w, p(i))\right| \leq m-k\right]$ and $\varphi_{k}\left(p_{-i}\right) \cap \beta_{k}(p(i))=\emptyset$ and $\varphi_{k}^{-}\left(p_{-i}\right) \cap \beta_{k}(p(i))=\emptyset$.

In this case $\varphi_{k}(p)=\{w\}$. Let $b=\beta\left(p(i)_{\mid \varphi_{k}^{-}\left(p_{-i}\right)}\right)$. Then $(b, w) \in p(i)$ since $\varphi_{k}^{-}\left(p_{-i}\right) \nsubseteq L(w, p(i))$. Construct an $i$-deviation $q$ of $p$ such that $\varphi_{k}^{-}\left(p_{-i}\right) \cap$ $\beta_{k}(q(i))=\{b\}$. Then $\varphi_{k}(q)=\{b, w\} \succ_{p(i)}\{b\}=\varphi_{k}(p)$.

D. Cases I(i) \& II(i) \& III(iii) \& IV(ii) and I(i) \& II(ii) \& III(iii) \& IV(ii). These cases are covered by the assumptions: $\left[\varphi_{k}\left(p_{-i}\right)=\{w\}\right.$ for some $w \in A$ and $\varphi_{k}^{-}\left(p_{-i}\right) \not \subset L(w, p(i))$ and $\left.\left|\varphi_{k}^{-}\left(p_{-i}\right) \cap L(w, p(i))\right| \leq m-k\right]$ and $\varphi_{k}\left(p_{-i}\right) \subseteq$ $\beta_{k}(p(i))$.

In this case, $\varphi_{k}(p)=\{w\}$. We can construct an $i$-deviation $q$ of $p$ such that $\varphi_{k}^{-}\left(p_{-i}\right) \cap L(w, p(i)) \subseteq A \backslash \beta_{k}(q(i))$. Then $\varphi_{k}(q)=\{w\} \cup\left[\varphi_{k}^{-}\left(p_{-i}\right) \cap \beta_{k}(q(i))\right]$. The set $\varphi_{k}^{-}\left(p_{-i}\right) \cap \beta_{k}(q(i))$ is non-empty and contains only points $x$ with $(x, w) \in$ $p(i)$. Hence, $\varphi_{k}(q) \succ_{p(i)} \varphi_{k}(p)$.

Proof of Corollary 5.4 For $2 \leq k \leq(m+1) / 2$ we have

$$
\eta(m, k)=\frac{m ! k^{2}[(m-k) !]^{2}}{(m-2 k+1) !}
$$


by Theorem 5.3. By a few elementary computations we obtain for $2 \leq k \leq(m+$ 1) $/ 2-1$ :

$$
\begin{aligned}
& \eta(m, k)<[>] \eta(m, k+1) \\
& \quad \Leftrightarrow(m-k)^{2} k^{2}<[>](k+1)^{2}(m-2 k+1)(m-2 k) .
\end{aligned}
$$

Denote $A:=(k+1)^{2}(m-2 k+1)(m-2 k)$, then $A>(k+1)^{2}(m-2 k)^{2}$ and this latter expression is at least as large as $(m-k)^{2} k^{2}$ if and only if $k^{2}+2 k-m \leq 0$, which, in turn, holds if and only if $k \leq \sqrt{m+1}-1$. Thus, by $(*)$,

$$
k \leq \underline{k}:=\sqrt{m+1}-1 \Rightarrow \eta(m, k)<\eta(m, k+1) .
$$

Similarly, $A<(k+1)^{2}(m-2 k+1)^{2}$ and this latter expression is not larger than $(m-k)^{2} k^{2}$ if and only if $k^{2}+k-(m+1) \geq 0$, which, in turn, holds if and only if $k \geq \frac{1}{2} \sqrt{1+4(m+1)}-\frac{1}{2}$. Thus, by $(*)$,

$$
k \geq \bar{k}:=\frac{1}{2} \sqrt{1+4(m+1)}-\frac{1}{2} \Rightarrow \eta(m, k)>\eta(m, k+1) .
$$

It is straightforward to derive that $\underline{k}<\bar{k}<(m+1) / 2$ and $\bar{k}-\underline{k}<1$. Now statement (a) follows by taking $k^{*}=\lfloor\underline{k}\rfloor+1$ or $k^{*} \in\{\lfloor\underline{k}\rfloor+1,\lfloor\underline{k}\rfloor+2\}$, depending on the exact values of $\underline{k}$ and $\bar{k}$.

For $m-1 \geq k>(m+1) / 2$ we have

$$
\eta(m, k)=\frac{m ! k ! k !}{(2 k-m) !}
$$

by Theorem 5.3. To go from $\eta(m, k)$ to $\eta(m, k+1)$ we multiply by a factor $(k+$ $1)^{2} /(2 k-m+2)(2 k-m+1)$, which is larger than 1 since $k+1 \geq 2 k-m+2$. This proves statement $(b)$.

To show statement (c) we have to show $\alpha(m, k)<1$ for all $2 \leq k \leq(m+1) / 2$, where $\alpha(m, k)=\eta(m, k) / \eta(m, m-1)$. By a simple computation we derive

$$
\alpha(m+1, k)=\alpha(m, k) \cdot \frac{(m+1-k)^{2}}{m^{2}(m-2 k+2)} .
$$

Since $(m+1-k)^{2}<m^{2}$ and $m-2 k+2 \geq 1$, this implies $\alpha(m+1, k)<\alpha(m, k)$. Hence, to show $\alpha(m, k)<1$ for all $2 \leq k \leq(m+1) / 2$, it is sufficient to show $\alpha(2 k+1, k)<1$ for all $k \geq 2$. We show this by induction on $k$. For $k=2$ we have $\alpha(5,2)=3 / 4<1$. Now assume $\alpha(2 k+1, k)<1$ then it is sufficient to show $\alpha(2 k+3, k+1)<1$. To show this, by straightforward computation we have

$$
\alpha(2 k+3, k+1)=\alpha(2 k+1, k) \cdot \frac{(k+2)^{2}}{2 k \cdot(2 k+1)} .
$$


By the induction hypothesis and since $(k+2)^{2}<2 k \cdot(2 k+1)$ for $k \geq 2$, we obtain $\alpha(2 k+3, k+1)<1$.

Proof of Proposition 6.2 Let $s$ be an arbitrary scoring vector. We will prove the proposition for the variance $\sigma^{2}(s)$, and first show that for $\sigma^{2}(s)$ to be maximal every coordinate of $s$ has to be equal to 0 or 1 . Let $j \in\{2, \ldots, m-1\}$ be arbitrary and write

$$
\sigma^{2}(s)=\frac{1}{m}\left[\sum_{i \neq j} s_{i}^{2}+s_{j}^{2}-m\left(\frac{\sum_{i \neq j} s_{i}+s_{j}}{m}\right)^{2}\right] .
$$

Differentiating with respect to $s_{j}$, we obtain after simplification:

$$
\frac{d \sigma^{2}(s)}{d s_{j}}=\frac{2(m-1)}{m^{2}}\left[s_{j}-\frac{\sum_{i \neq j} s_{i}}{m-1}\right] .
$$

This implies $\frac{d \sigma^{2}(s)}{d s_{j}}>(=,<) 0$ exactly when $s_{j}>(=,<) \frac{\sum_{i \neq j} s_{i}}{m-1}$. In turn, this implies that with respect to $s_{j}$ the variance $\sigma^{2}(s)$ is maximal for $s_{j}=0$ or $s_{j}=1$.

Second, let $\sigma^{2}(k)$ denote the variance of the scoring vector associated with the $k$-approval rule. Then it is simple to verify that $\sigma^{2}(k)=(1 / m)\left[k-k^{2} / m\right]$, which implies that $\sigma^{2}(k)$ is maximal exactly if $k \in\{(m-1) / 2,(m+1) / 2\}$ for $m$ odd and $k=m / 2$ for $m$ even.

The proof is complete by combining the two arguments.

\section{References}

Aleskerov F, Kurbanov E (1999) Degree of manipulability of social choice procedures. In: Proceedings of the third international meeting of the society for the advancement of economic theory. Springer, Berlin

Barberà S, Dutta B, Sen A (2001) Strategy-proof social choice correspondences. J Econ Theory 101:374394

Brams SJ (2010) Preface. In: Laslier JF, Sanver MR (eds) Handbook on approval voting. Springer, Heidelberg, pp vii-ix

Brams SJ, Fishburn PC (1983) Approval voting. Birkhauser, Boston

Brams SJ, Fishburn PC (2005) Going from theory to practice: the mixed success of approval voting. Soc Choice Welf 25:457-474 (Reprinted: In: Laslier JF, Sanver MR (eds) (2010) Handbook on approval voting. Studies in social choice and welfare, Springer, Berlin)

Campbell DE, Kelly JS (2009) Gains from manipulating social choice rules. Econ Theory 40:349-371

Diss M, Merlin V, Valognes F (2010) On the Condorcet efficiency of approval voting and extended scoring rules for three alternatives. In: Laslier JF, Sanver MR (eds) Handbook on approval voting. Springer, Berlin, pp 255-283

Fristrup P, Keiding H (1998) Minimal manipulability and interjacency for two-person social choice functions. Soc Choice Welf 15:455-467

Gehrlein WV, Lepelley D (1998) The Condorcet efficiency of approval voting and the probability of electing the Condorcet loser. J Math Econ 29:271-283

Gibbard A (1973) Manipulation of voting schemes: a general result. Econometrica 41:587-602

Kelly JS (1988) Minimal manipulability and local strategy-proofness. Soc Choice Welf 5:81-85

Kelly JS (1989) Interjacency. Soc Choice Welf 6:331-335

Laslier JF, Sanver MR (eds) (2010) Handbook on approval voting. Studies in social choice and welfare. Springer, Berlin 
Lepelley D, Mbih B (1994) The vulnerability of four social choice functions to coalitional manipulation of preferences. Soc Choice Welf 11:253-265

Maus S, Peters H, Storcken T (2007) Minimal manipulability: unanimity and nondictatorship. J Math Econ 43:675-691

Pritchard G, Wilson MC (2009) Asymptotics of the minimum manipulating coalition size for positional voting rules under impartial culture behaviour. Math Soc Sci 58:35-57

Saari D (1990) Susceptibility to manipulation. Public Choice 64:21-41

Sanver MR (2010) Approval as an intrinsic part of preference. In: Laslier JF, Sanver MR (eds) Handbook on approval voting. Springer, Berlin, pp 469-481

Satterthwaite M (1975) Strategy-proofness and Arrow's conditions: existence and correspondence theorems for voting procedures and social welfare functions. J Econ Theory 10:187-217

Young P (1975) Social choice scoring functions. SIAM J Appl Math 27:824-838 